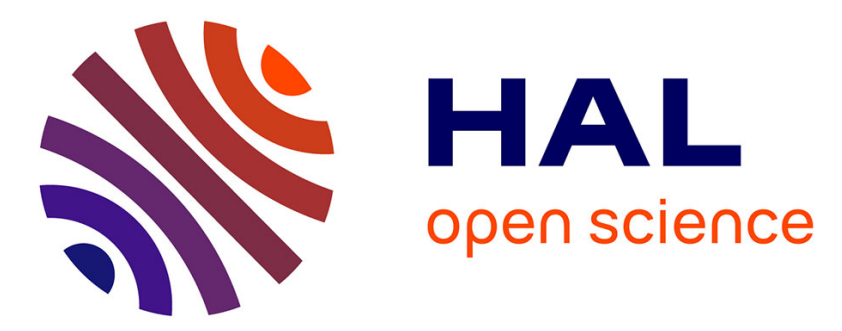

\title{
First lifetime investigations of $N>82$ iodine isotopes: The quest for collectivity
}

G. Häfner, R. Lozeva, H. Naïdja, M. Lebois, N. Jovančević, D. Thisse, D. Etasse, R.L. Canavan, M. Rudigier, J.N. Wilson, et al.

\section{- To cite this version:}

G. Häfner, R. Lozeva, H. Naïdja, M. Lebois, N. Jovančević, et al.. First lifetime investigations of $N>82$ iodine isotopes: The quest for collectivity. Phys.Rev.C, 2021, 104 (1), pp.014316. 10.1103/PhysRevC.104.014316 . hal-03312933

\section{HAL Id: hal-03312933 https://hal.science/hal-03312933}

Submitted on 7 Oct 2021

HAL is a multi-disciplinary open access archive for the deposit and dissemination of scientific research documents, whether they are published or not. The documents may come from teaching and research institutions in France or abroad, or from public or private research centers.
L'archive ouverte pluridisciplinaire HAL, est destinée au dépôt et à la diffusion de documents scientifiques de niveau recherche, publiés ou non, émanant des établissements d'enseignement et de recherche français ou étrangers, des laboratoires publics ou privés. 


\section{First lifetime investigations of $N>82$ iodine isotopes: The quest for collectivity}

G. Häfner, ${ }^{1,2}$ R. Lozeva $\odot,{ }^{1, *}$ H. Naïdja, ${ }^{3}$ M. Lebois, ${ }^{1}$ N. Jovančević, ${ }^{1}$ D. Thisse,,${ }^{1}$ D. Etasse, ${ }^{4}$ R. L. Canavan,,${ }^{5,6}$ M. Rudigier,,${ }^{5,7}$ J. N. Wilson, ${ }^{1}$ E. Adamska, ${ }^{8}$ P. Adsley,${ }^{5}$ A. Algora,${ }^{9}$ M. Babo, ${ }^{1}$ K. Belvedere, ${ }^{5}$ J. Benito, ${ }^{10}$ G. Benzoni, ${ }^{11,12}$ A. Blazhev, ${ }^{2}$ A. Boso, ${ }^{6}$ S. Bottoni, ${ }^{11,12}$ M. Bunce,${ }^{6}$ R. Chakma, ${ }^{1}$ N. Cieplicka-Oryńczak, ${ }^{13}$ S. M. Collins,${ }^{6}$ M. L. Cortés, ${ }^{14,15}$ P. J. Davies,${ }^{16}$ C. Delafosse, ${ }^{1}$ M. Fallot, ${ }^{17}$ L. M. Fraile,${ }^{10}$ R.-B. Gerst, ${ }^{2}$ D. Gjestvang,${ }^{18}$ V. Guadilla,${ }^{17}$ K. Hauschild,${ }^{1}$ C. Henrich, ${ }^{7}$ I. Homm, ${ }^{7}$ F. Ibrahim, ${ }^{1}$ E. W. Iskra, ${ }^{11,13}$ S. Jazwari, ${ }^{5,6}$ A. Korgul, ${ }^{8}$ P. Koseoglou, ${ }^{7}$ Th. Krölll, ${ }^{7}$ T. Kurtukian-Nieto, ${ }^{19}$ L. Le-meur, ${ }^{17}$ S. Leoni, ${ }^{11,12}$ J. Ljungvall, ${ }^{19}$ A. Lopez-Martens, ${ }^{19}$ L. Matthieu, ${ }^{19}$ K. Miernik, ${ }^{8}$ J. Nemer, ${ }^{1}$ S. Oberstedt, ${ }^{20}$ W. Paulsen, ${ }^{18}$ M. Piersa-Siłkowska ${ }^{8}$ Y. Popovitch, ${ }^{1}$ C. Porzio, ${ }^{11,12,21}$ L. Qi ${ }^{1}$ D. Ralet,${ }^{22,1}$ P. H. Regan,,${ }^{5,6}$ D. Reygadas Tello, ${ }^{23}$ K. Rezynkina, ${ }^{24,25}$ V. Sanchez-Tembleque, ${ }^{10}$ C. Schmitt, ${ }^{25}$ P.-A. Söderström, ${ }^{7,26}$ C. Sürder ${ }^{7}$ G. Tocabens, ${ }^{1}$ V. Vedia, ${ }^{10}$ D. Verney, ${ }^{1}$ N. Warr, ${ }^{2}$ B. Wasilewska, ${ }^{13}$ J. Wiederhold, ${ }^{7}$ M. S. Yavahchova, ${ }^{27}$ F. Zeiser, ${ }^{18}$ and S. Ziliani ${ }^{11,12}$

${ }^{1}$ Univeristé Paris-Saclay, IJCLab, CNRS/IN2P3, F-91405 Orsay, France

${ }^{2}$ Institut für Kernphysik, Universität zu Köln, D-50937 Köln, Germany

${ }^{3}$ Universté Constantine 1, LPMS, route Ain El Bey DZ-25000, Constantine, Algeria ${ }^{4}$ LPC Caen, CNRS/IN2P3, F-14000 Caen, France

${ }^{5}$ Department of Physics, University of Surrey, Guilford, GU2 7XH, United Kingdom

${ }^{6}$ National Physical Laboratory, Teddington, Middlesex, TW11 OLW, United Kingdom

${ }^{7}$ Technische Universität Darmstadt, Insitut für Kernphysik, D-64289, Darmstadt, Germany

${ }^{8}$ Faculty of Physics, University of Warsaw, PL 02-093, Warsaw, Poland

${ }^{9}$ Instituto de Fisica Corpuscular, CSIC-Universidad de Valencia, E-46071 Valencia, Spain

${ }^{10}$ Grupo de Física Nuclear \& IPARCOS, Universidad Complutense de Madrid, E-28040 Madrid, Spain

${ }^{11}$ Dipartimento di Fisica, Iniversitá degli Studi di Milano, I-20133 Milano, Italy

${ }^{12}$ INFN Sezione di Milano, Via Celoria 16, I-20133 Milano, Italy

${ }^{13}$ Institute of Nuclear Physics Polish Academy of Sciences, PL-31342 Krakow, Poland

${ }^{14}$ RIKEN Nishina Center, 2-1 Hirosawa, Wako, Saitama 351-0198, Japan

${ }^{15}$ INFN Laboratori Nazionali di Legnaro, Viale dell Universitá 2, I-35020 Legnaro, Italy

${ }^{16}$ Department of Physics, University of Manchester, Oxford Road, Manchester M 13 9PL, United Kingdom

${ }^{17}$ Subatech/Ecole des Mines, CNRS/IN2P3, Université de Nantes, F-44307 Nantes, France

${ }^{18}$ University of Oslo, Department of Physics, NO-0316 Oslo, Norway

${ }^{19}$ CENBG Bordeaux, CNRS/IN2P3, Université de Bordeaux, BP 120, F-33175 Gradignan, France

${ }^{20}$ European Commission, Joint Research Centre (JRC), BE-2440 Geel, Belgium

${ }^{21}$ TRIUMF, 4004 Wesbrook Mall, Vancouver, British Columbia V6T 2A3, Canada

${ }^{22}$ Grand Accelérateur National d'Ions Lourds, CEA/DSM-CNRS/IN2P3, F-14076 Caen Cedex, France

${ }^{23}$ Insitut Laue-Langevin, F-38042 Grenoble Cedex, France

${ }^{24}$ Insitute for Nuclear and Radiation Physics, KU Leuven, BE-3000 Leuven, Belgium

${ }^{25}$ Université de Strasbourg, IPHC, CNRS/IN2P3, F-67037 Strasbourg, France

${ }^{26}$ Extreme Light Infrastructure - Nuclear Physics, RO-077125 Bucharest-Magurele, Romania

${ }^{27}$ Institute for Nuclear Research and Nuclear Energy, Bulgarian Academy of Sciences (BAS), BG-1784 Sofia, Bulgaria

(Received 9 March 2021; revised 31 May 2021; accepted 25 June 2021; published 19 July 2021)

We report on spectroscopic information and lifetime measurements in the neutron-rich ${ }^{135,137,139}$ I isotopes. This is the first lifetime data on iodine isotopes beyond $N=82$. Excited states were populated in fast neutroninduced fission of ${ }^{238} \mathrm{U}$ at the ALTO facility of IJCLab with the LICORNE neutron source and detected using the hybrid $v$-ball spectrometer. The level schemes of the ${ }^{135,137,139} \mathrm{I}$ isotopes are revised in terms of excited states with up to maximum spin-parity of $\left(33 / 2^{+}\right)$, populated for the first time in fast neutron-induced fission. We provide first results on the lifetimes of the $\left(9 / 2_{1}^{+}\right)$and $\left(13 / 2_{1}^{+}\right)$states in ${ }^{137} \mathrm{I}$ and ${ }^{139} \mathrm{I}$, and the $\left(17 / 2_{1}^{+}\right)$state in ${ }^{137} \mathrm{I}$. In addition, we give upper lifetime limits for the $\left(11 / 2_{1}^{+}\right)$states in ${ }^{135-139} \mathrm{I}$, the $\left(15 / 2_{1}^{+}\right)$ state in ${ }^{137} \mathrm{I}$, the $\left(17 / 2_{1}^{+}\right)$state in ${ }^{139} \mathrm{I}$, and reexamine the $\left(29 / 2_{1}^{+}\right)$state in ${ }^{137} \mathrm{I}$. The isomeric data in ${ }^{135} \mathrm{I}$ are reinvestigated, such as the previously known $\left(15 / 2_{1}^{+}\right)$and $\left(23 / 2_{1}^{-}\right)$isomers with $T_{1 / 2}$ of $1.64(14)$ and $4.6(7)$ ns, respectively, as obtained in this work. The new spectroscopic information is compared to that from spontaneous or thermal-neutron induced fission and discussed in the context of large scale shell-model (LSSM) calculations

*radomira.lozeva@ijclab.in2p3.fr 
for the region beyond ${ }^{132} \mathrm{Sn}$, indicating the behavior of collectivity for the three valence-proton iodine chain with $N=82,84,86$.

DOI: 10.1103/PhysRevC.104.014316

\section{INTRODUCTION AND MOTIVATION}

The study of nuclei with few valence particles outside the doubly magic ${ }^{132} \mathrm{Sn}$ core provides important information on two-body matrix elements to test realistic interactions employed in nuclear shell-model calculations. The development of collectivity outside the ${ }^{132} \mathrm{Sn}$ core is of current interest [1-9] and experimental findings provide a valuable input for state-of-the-art nuclear theories. The high degree of regularity in excitation energies along isotonic chains for nuclei "northeast" of ${ }^{132} \mathrm{Sn}$ project the overall behavior in that region. However, such experimental information is insufficient for understanding their nuclear structure and the development of collectivity. Thus, more experimental data are required from studying individual nuclei. The $Z=53$ iodine isotopes have three valence protons outside the doubly magic core. The study of odd- $Z$ nuclei, in particular of the iodine isotopes, reveals key information on the proton-neutron interaction and their associated coupling schemes far off the valley of stability. The three valence protons acting as a cluster [10] has been suggested to describe some of the features in the iodine isotopes [11,12]. It was expected that such three-proton configurations would result in a more complex level structure in the odd-odd iodine isotopes, with such an effect also in their odd-even neighbors; however, their particular role has not been identified so far $[7,13,14]$.

Above $Z=50$, the evolution of the low-lying proton $\pi\left(1 d_{5 / 2}\right)$ and $\pi\left(0 g_{7 / 2}\right)$ orbitals as a function of neutron number, $N$, is of particular interest. An indication for the inversion of those orbitals was observed in a long-lived $\left(6^{-}\right)$isomer in the ${ }^{140} \mathrm{Sb}$ nucleus [15]. In the odd-odd ${ }^{136,138}$ I isotopes longlived isomers $[13,16,17]$ were suggested to result from the drastic lowering in the position of the $\pi\left(1 d_{5 / 2}\right)$ orbital $[13,18]$. Recent results on ${ }^{136} \mathrm{I}$, studied in various reactions, identify a relatively large energy spacing between the two proton orbitals that decreases systematically with increasing $N$ [7].

For the semimagic ${ }^{135}$ I nucleus, numerous measurements on excited states were performed in Refs. [19,20] and references therein. The ${ }^{137} \mathrm{I}$ isotope was examined via $\beta$ decay of ${ }^{137} \mathrm{Te}$ [21] and spontaneous fission of ${ }^{248} \mathrm{Cm}$ [22] and ${ }^{252} \mathrm{Cf}$ [23]. The study of yrast states in ${ }^{137} \mathrm{I}$, populated in spontaneous fission of ${ }^{248} \mathrm{Cm}$, revealed the presence of both $\pi\left(0 g_{7 / 2}^{3}\right)$ and $\pi\left(0 g_{7 / 2} 1 d_{5 / 2}\right)$ proton couplings [22]. Further investigations [23] have shown a strong correlation between the low-lying yrast states in the odd- $Z N=84$ isotones and the excitation energy $E_{x}\left(2_{1}^{+}\right)$in the respective even- $Z$ isotones. For the ${ }^{139}$ I nucleus, the only information on yrast states was measured in a spontaneous fission experiment [14], reporting on a similar excitation scheme as its $A-2$ neighbor [24,25]. Recently, excited states in ${ }^{139} \mathrm{I}$ were also populated in the $\beta$-delayed neutron decay of ${ }^{140} \mathrm{Te}$ [26].

While these neutron-rich iodine nuclei have been studied extensively, lifetime information on the exotic ones has only been reported for ${ }^{135} \mathrm{I}$ having $N=82$ [20]. In the measurement of this three-valence proton nucleus ${ }^{135} \mathrm{I}$, seniority relations were tested and the inferred $B\left(E 2 ; 15 / 2^{+} \rightarrow 11 / 2^{+}\right)$transition strength was described in a single orbit $\pi\left(0 g_{7 / 2}^{3}\right)$ approximation [20]. With the advent and development of large hybrid arrays employing high-purity germanium (HPGe) detectors and $\mathrm{LaBr}_{3}(\mathrm{Ce})$ scintillators, the well established fast-timing technique can be applied to measure lifetimes of low-lying excited states $[27,28]$ and to largely extend such investigations.

It can be expected that, with the increase of $N$ and the increase of valence nucleons, the collective excitations dominate over the single-particle modes, thus resulting in lifetimes of low-lying yrast states in the ns/sub-ns range. This is in the range of the fast electronic timing technique. From the lifetime information reduced transition probabilities can be extracted, giving valuable insights into the development of collectivity and its interplay with the single-particle excitation nature of states outside the ${ }^{132} \mathrm{Sn}$ core.

This work focuses on the population of neutron-rich iodine isotopes in fast neutron-induced fission of ${ }^{238} \mathrm{U}$ and reports for the first time on lifetime measurements of low-lying excited states in iodine nuclei with $N>82$. Starting from ${ }^{135} \mathrm{I}$, as a test case with a known isomer, the lifetime information is extended to the ${ }^{137,139}$ I isotopes. The study of fission fragments and the population of intermediate and high-spin states is also of great importance for future reactor generations, while knowledge of prompt $\gamma$-ray emission in fission fragments is related to the heating problem in nuclear energy applications $[29,30]$.

\section{EXPERIMENT AND ANALYSIS}

The ${ }^{135,137,139}$ I nuclei were produced in fast neutroninduced fission of ${ }^{238} \mathrm{U}$ as part of the $v$-ball fission campaign. A schematic description of the setup is shown in Fig. 1(a). The LICORNE neutron source was used to produce fast neutrons via the $p\left({ }^{7} \mathrm{Li},{ }^{7} \mathrm{Be}\right) n$ reaction [31]. The primary ${ }^{7} \mathrm{Li}$ beam provided by the Tandem accelerator of the ALTO facility was pulsed with a repetition time of $400 \mathrm{~ns}$ and a width of $\sigma \sim$ $0.85 \mathrm{~ns}$. This allowed the reconstruction of prompt-delayed coincidence events and time-reference measurements with respect to the beam. The average neutron energy was chosen according to the fission cross section to be $\approx 1.7 \mathrm{MeV}$ and the neutrons were kinematically guided onto a thick ${ }^{238} \mathrm{U}$ target to induce fission. The target was composed of five disks of uranium arranged in an $8 \mathrm{~cm}$ long cylindrical aluminum casing with a total mass of $81 \mathrm{~g}$. This shape was chosen to minimize self-absorption of low-energetic $\gamma$ rays. The target was located in the middle of the array as shown in Fig. 1(a).

The $\gamma$ rays from fission fragments were detected with the hybrid $v$-ball spectrometer [33]. The array consisted of highpurity germanium (HPGe) and $\mathrm{LaBr}_{3}(\mathrm{Ce})$ detectors placed in four rings [R1 to R4 in Fig. 1(a)] around the target. The 


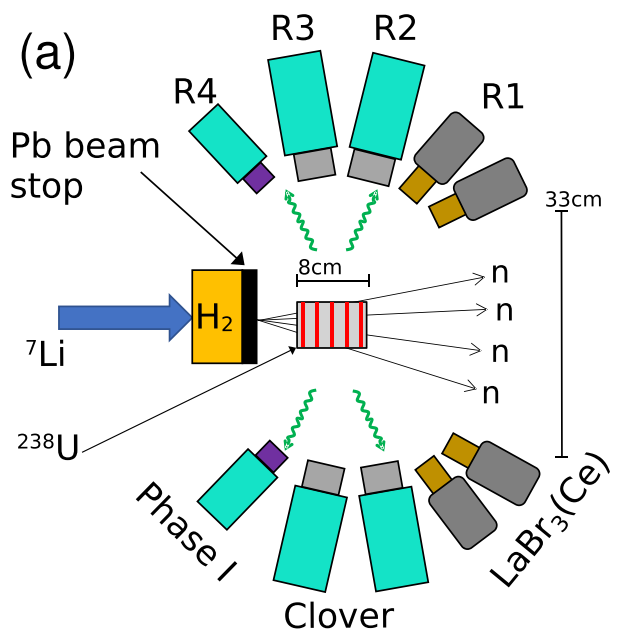

(b)

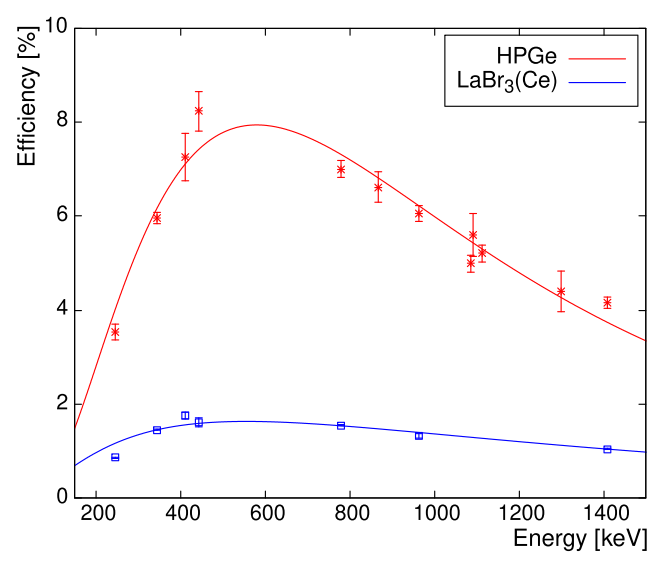

FIG. 1. (a) Schematic representation of the experimental setup. The neutrons are produced in the ${ }^{7} \operatorname{Li}(p, n){ }^{7} \mathrm{Be}$ reaction and are focused on the ${ }^{238} \mathrm{U}$ target to induce fission. The $\gamma$ rays are detected by the $v$-ball array consisting of $20 \mathrm{LaBr}_{3}(\mathrm{Ce})$ detectors (ring 1), $24 \mathrm{HPGe}$ Clover detectors (rings 2 and 3), and ten HPGe Phase-I detectors (ring 4). Adapted from Ref. [32]. (b) HPGe (red) and LaBr ${ }_{3}(\mathrm{Ce})$ (blue) efficiency curves. The data points were obtained using a ${ }^{152} \mathrm{Eu}$ source and corrected for self-absorption effects. For details see text.

first ring $(\mathrm{R} 1)$ was composed of ten 1.5 in. $\times 2$ in. cylindrical and ten 1 in. $\times 1.5$ in. $\times 2$ in. conical $\mathrm{LaBr}_{3}(\mathrm{Ce})$ scintillators. Each of the second and third rings (R2, R3) consisted of 12 HPGe clover type detectors. The ball had an additional ring (R4) of ten coaxial phase-I HPGe detectors. All HPGe detectors were equipped with bismuth germanate (BGO) shields to reduce background from Compton scattering. The typical energy resolution for the summed-up spectra from the HPGe detectors was measured to be about $2.5 \mathrm{keV}$ at $1.3 \mathrm{MeV}$, whereas for the clover and the phase-I detectors these resolutions are of the order of $1.8 \mathrm{keV}$ and $2.9 \mathrm{keV}$, respectively $[28,33]$. The time resolution for the $\mathrm{HPGe}$ and $\mathrm{LaBr}_{3}(\mathrm{Ce})$ detectors measured with a ${ }^{60} \mathrm{Co}$ source was about $13 \mathrm{~ns}$ and $\sim 200 \mathrm{ps}$, respectively [33]. The $\gamma$-ray detection efficiency could be deduced from a ${ }^{152} \mathrm{Eu}$ source measurement which is corrected for self absorption due to the target geometry using the GEANT4 simulation toolkit [34]. The use of a thick actinide target resulted in a drop of the $\gamma$-ray efficiency for energies around $300 \mathrm{keV}$ or below. For higher energies, e.g., 1.3 MeV, the efficiency amounts to about 4.1(2)\% for HPGe and $0.7(1) \%$ for $\operatorname{LaBr}_{3}(\mathrm{Ce})$ detectors [28]. These efficiency curves are shown in Fig. 1(b).

The data were recorded in a triggerless mode using the digital acquisition system FASTER [35] which is able to handle a large number of channels and detector types at the same time. Two different types of digitizers were used: $125 \mathrm{MHz}, 14-$ bit analog-to-digital converters (ADCs) for HPGe and BGO detectors and $500 \mathrm{MHz}, 12$-bit charge-to-digital converters (QDCs) for $\mathrm{LaBr}_{3}(\mathrm{Ce})$ scintillators [33]. The average rates of the HPGe detectors were in the order of 5-7 kHz. Energy and time information of all detectors was recorded, allowing for detailed calorimetry studies and full event multiplicity reconstruction (see, for example, Refs. [33,36]). Over the course of $216 \mathrm{~h}$ measurement time, approximately $12.5 \mathrm{~TB}$ of raw data were acquired. After energy calibration, time alignment and gain matching on all detectors, fission events were reconstructed based on different multiplicity conditions.
First, a coarse trigger condition was applied based on either two HPGe modules (after Compton suppression and addback) firing within one beam pulsation (400 ns) or two $\mathrm{LaBr}_{3}(\mathrm{Ce})$ detectors firing around the prompt width (15 ns) of the beam pulse. This was done to suppress low-multiplicity events such as inelastic scattering of the ${ }^{7} \mathrm{Li}$ primary beam or neutrons. Additional background was generated by reactions of the primary beam with lead beam stopper [as shown in Fig. 1(a)].

A HPGe singles spectrum is shown in Fig. 2, where some of these contaminants are marked together with transitions from well-populated fission fragments $\left({ }^{140} \mathrm{Xe}\right.$ and $\left.{ }^{134} \mathrm{Te}\right)$ and isotopes of interest $\left({ }^{135,137} \mathrm{I}\right)$. Further constraints based on the total modular multiplicity $M$ [HPGe module and $\mathrm{LaBr}_{3}(\mathrm{Ce})$ together] can be used to improve the peak-to-total ratio of the $\gamma$ rays from the fission fragments of interest. This is visualized in the inset of Fig. 2, showing a HPGe energy projection for

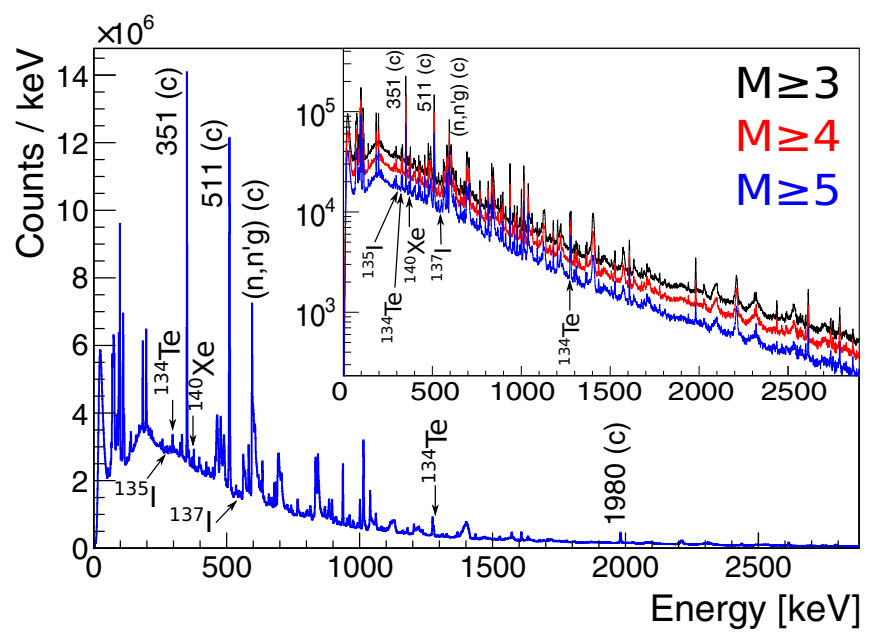

FIG. 2. HPGe singles spectrum labeled with contaminants ("c") and transitions from different fission fragments. The inset shows an energy projection with different multiplicity conditions $M$. 

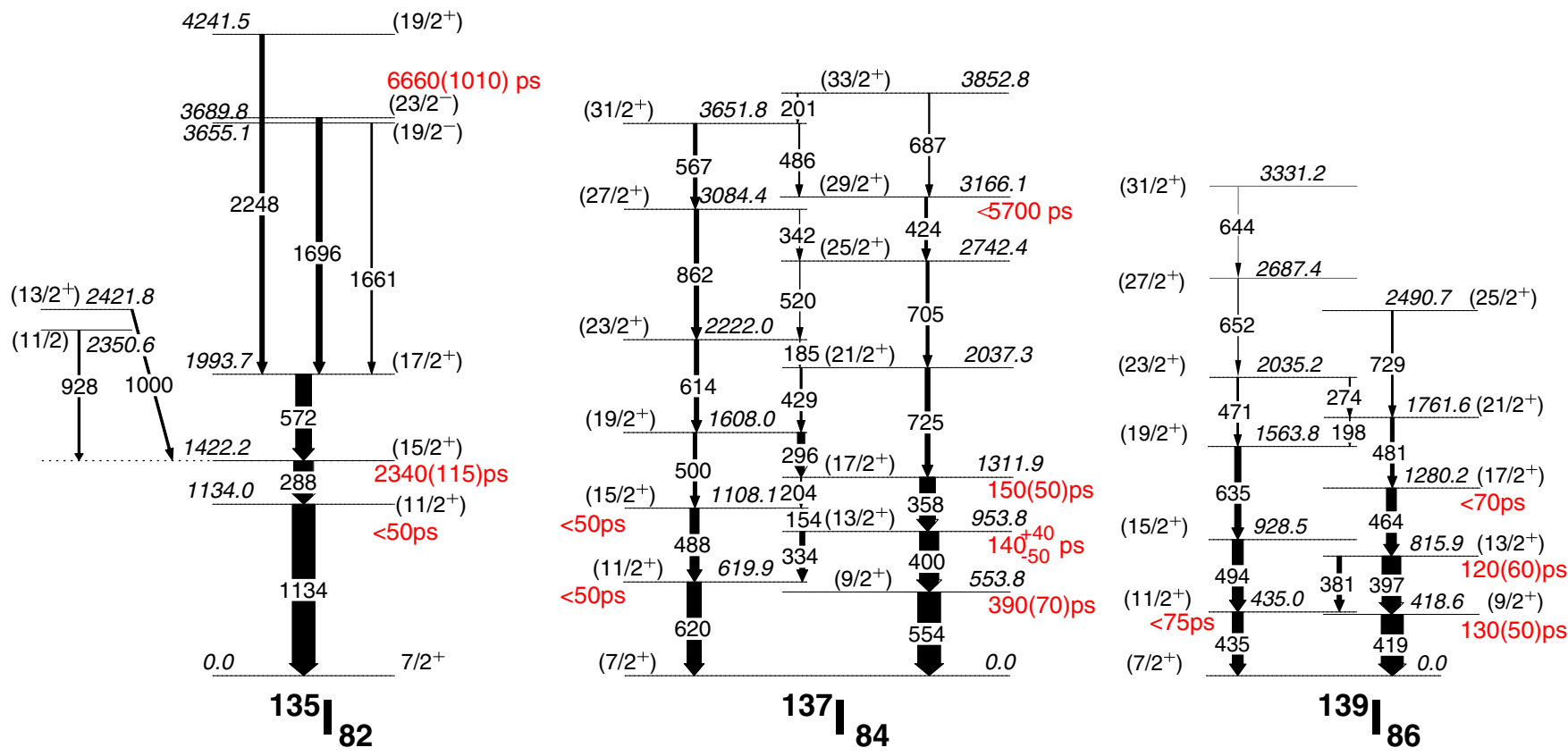

FIG. 3. Level schemes for the ${ }^{135,137,139}$ I isotopes as observed in this work. The arrow widths correspond to the relative $\gamma$-ray intensities. Where applicable, states are labeled with their lifetime as measured in this work.

different total multiplicity conditions, where, e.g., $M \geqslant 3$ is the minimum required for our type of analysis. As can be seen in this figure, the background level is significantly reduced by selecting higher multiplicity. Therefore, when needed, these multiplicity conditions are used to appropriately select the transitions of interest with respect to the background. Overall, a lower multiplicity condition of $M \geqslant 5$ was proven to be useful in enhancing $\gamma$ rays from fission fragments and was also used in Ref. [32].

The time information is extracted in various ways, depending on the lifetime range of the excited state of interest. The HPGe time information with respect to the beam timing is used for measurements of lifetimes $\tau \gtrsim 5 \mathrm{~ns}$, limited by the time resolution of the HPGe detectors. For lifetimes around $1 \mathrm{~ns}$, the $\mathrm{LaBr}_{3}(\mathrm{Ce})$ beam timing information is used. The $\mathrm{LaBr}_{3}(\mathrm{Ce})$ time resolution, superior by more than an order of magnitude, does not contribute significantly to the width of the time profile. For lifetimes of $\tau<1 \mathrm{~ns}$, the $\mathrm{LaBr}_{3}(\mathrm{Ce})$ $\operatorname{LaBr}_{3}(\mathrm{Ce})$ time difference $(\Delta T)$ is measured, and has a prompt width of $\sigma \sim 0.3-0.6 \mathrm{~ns}$ depending on the energy range. $\mathrm{HPGe}-\mathrm{LaBr}_{3}(\mathrm{Ce})-\mathrm{LaBr}_{3}(\mathrm{Ce})$ cubes are created based on two $\operatorname{LaBr}_{3}(\mathrm{Ce})$ hits within the $15 \mathrm{~ns}$ prompt width in coincidence with a HPGe module. The time differences are constructed after selecting the nuclei of interest with one or two clean background-subtracted HPGe gates. Then, the data are sorted into symmetric $E_{\gamma}-E_{\gamma}-\Delta T$ cubes. Time difference measurements of decaying and feeding transitions result in the delayed time distribution, denoted as $\Delta T\left(E_{\text {decay }}, E_{\text {feeder }}\right)$. Whenever applicable, the time distributions are fitted with a convolution of a prompt Gaussian and an exponential decay. The knowledge of the prompt distribution as a function of energy is crucial for this technique and is investigated through known prompt transitions (see Fig. 1 of Ref. [28]). The resulting error of the lifetimes includes contributions from varying the fit region, the background component and the prompt width. These systematic components of the fit are carefully investigated by measuring previously known lifetimes. Thus, the main uncertainty in the results originates from statistical fluctuations, which for very exotic nuclei with low statistics may be significant.

\section{RESULTS}

\section{A. Population of ${ }^{135,137,{ }^{139}}$ I}

According to Ref. [37], the estimated fission yields for the isotopes of interest are $\sim 1.5 \%$ for ${ }^{135} \mathrm{I}, \sim 3.3 \%$ for ${ }^{137} \mathrm{I}$ and $\sim 2.4 \%$ for ${ }^{139} \mathrm{I}$. As the previously known $\left(5 / 2^{+}\right) \rightarrow$ $\left(7 / 2^{+}\right)$ground state transitions in the ${ }^{137,139}$ I isotopes are not observed in this work, their contribution is assumed to be negligible. The following relative yields are obtained: $Y\left({ }^{137} \mathrm{I} /{ }^{135} \mathrm{I}\right)=4.0(11)$ and $Y\left({ }^{137} \mathrm{I} /{ }^{139} \mathrm{I}\right)=1.5(8)$. While the latter ratio compares well the estimated yields for $A=137$ and 139 , the ${ }^{135}$ I nucleus seems to be produced about a factor of 2 less with a yield of about 0.9(2) \%. One may also note here the comparability in terms of $U$ fission yields to the evaluated data on $\mathrm{Cm}$ for the mass range $A=130-140[38,39]$.

From the measured $\gamma$-ray transitions in this work, the level schemes in Fig. 3 were obtained. Details on these transitions and the populated states can also be seen in Table I. The $\gamma$-ray intensities are normalized to the strongest transitions, namely $1134 \mathrm{keV}\left(11 / 2^{+}\right) \rightarrow 7 / 2^{+}$in ${ }^{135} \mathrm{I}, 554 \mathrm{keV}$ $\left(9 / 2^{+}\right) \rightarrow\left(7 / 2^{+}\right)$in ${ }^{137} \mathrm{I}$, and $419 \mathrm{keV}\left(9 / 2^{+}\right) \rightarrow\left(7 / 2^{+}\right)$in the ${ }^{139} \mathrm{I}$ isotope.

To determine the intensity ratio of the parallel ground state transitions in the ${ }^{137,139} \mathrm{I}$ isotopes, various gates from the ${ }_{39} \mathrm{Y}$ binary fission partners are used. For the strongest isotope, ${ }^{137} \mathrm{I}$, spins up to $J=33 / 2$ and an excitation energy of about 3.9 MeV could be populated. An excitation energy of up to 
TABLE I. Transitions associated with the ${ }^{135,137,139}$ I isotopes as observed in this work. Spins and parities are taken from Refs. [19,24,25]. Intensities are normalized to the strongest transitions and corrected for efficiency. Literature values are taken from spontaneous ${ }^{248} \mathrm{Cm}$ fission data from Refs. [14,22,41].

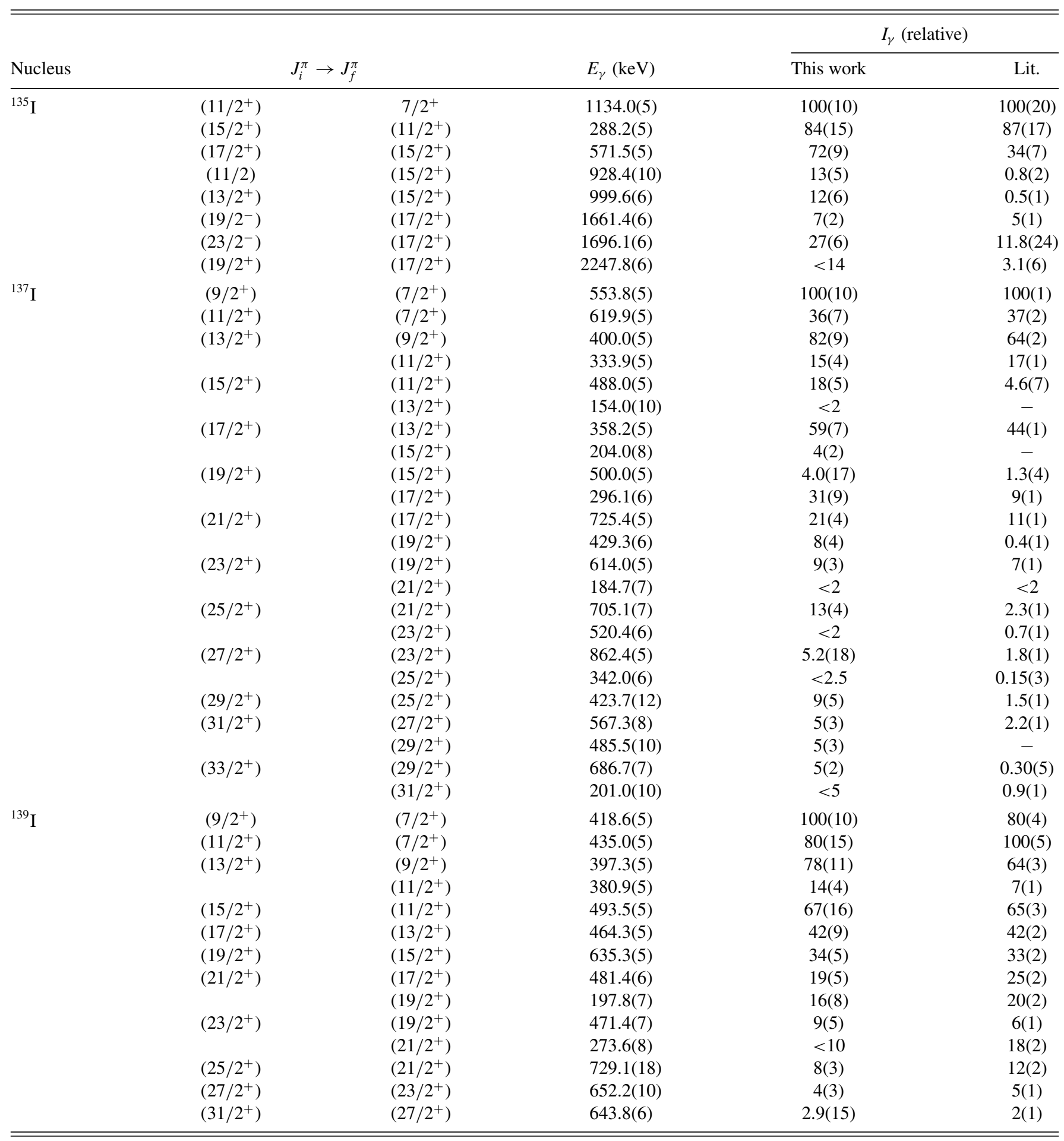

4.2 $\mathrm{MeV}$ is detected for the ${ }^{135} \mathrm{I}$ nucleus and a maximum spin of $J=23 / 2$. For ${ }^{139} \mathrm{I}$, only some of the previously known transitions are detected, corresponding to a maximum spin of $J=31 / 2$. Despite detection limitations such as low statistics and relatively high background in the $\gamma$-ray spectra, the population in fast neutron-induced fission provides valuable input on the population of angular momentum and excitation energy of fission fragments that may be exploited in future studies [40].

Figure 4 shows HPGe energy projections, gated on transitions in the ${ }^{137} \mathrm{I}$ and ${ }^{139} \mathrm{I}$ isotopes. The identification of the nuclei is possible despite the standard contaminations from $\left(n, n^{\prime} \gamma\right)$ reactions or the primary beam. In particular, the 358 $400-554 \mathrm{keV}$ cascade in ${ }^{137} \mathrm{I}$ and the $397-419 \mathrm{keV}$ cascade in 

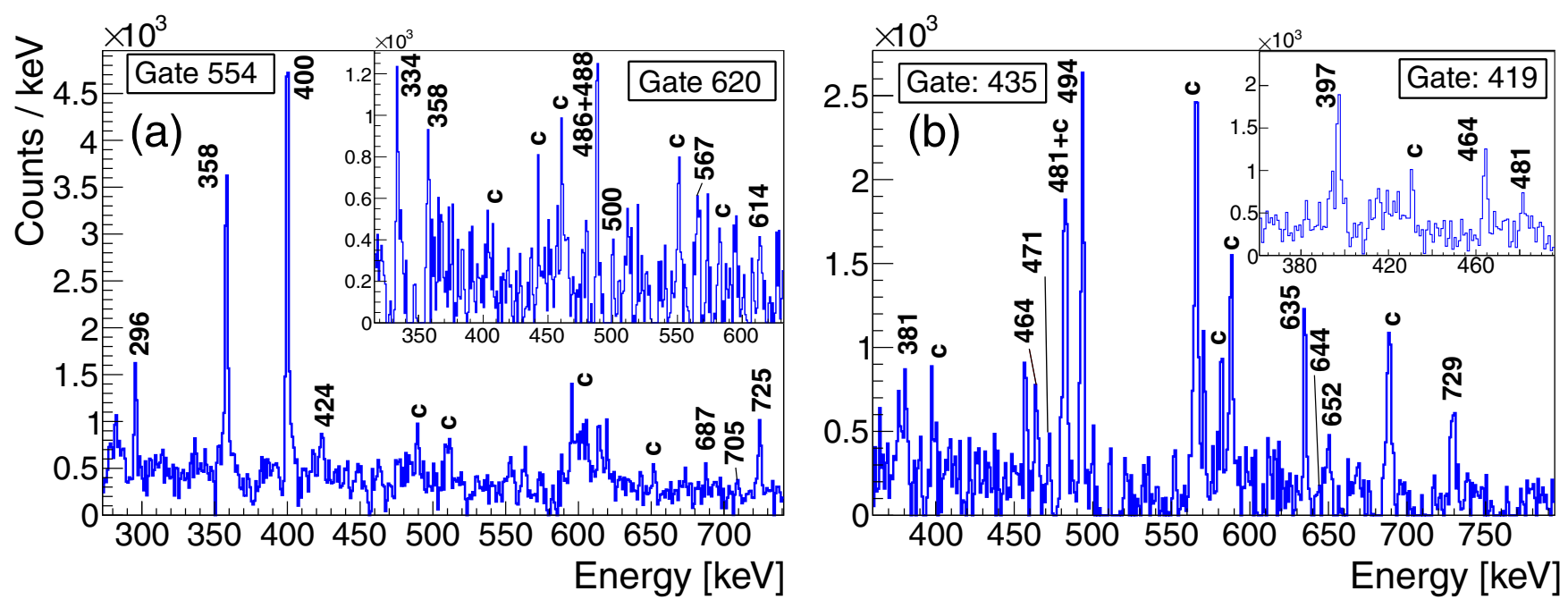

FIG. 4. HPGe energy projections after gating on ground state transitions in (a) ${ }^{137} \mathrm{I}$ and (b) ${ }^{139} \mathrm{I}$ as labeled. Transitions from the isotopes of interest are indicated by their respective energy, while those from the background (inelastic neutron scattering or fission binary partners) are labeled with "c".

${ }^{139}$ I are well identified. Furthermore, the heights of the peaks are comparable to the relative population of both isotopes as pointed out above.

The experimental values for relative $\gamma$-ray intensities are compared to data from previous ${ }^{248} \mathrm{Cm}$ spontaneous fission experiments in Table I. An overall consistency in the intensity pattern is observed, despite some deviations of more than $3 \sigma$, which may be attributed to the different reaction mechanisms. For ${ }^{135} \mathrm{I}$ there are two major differences. First, the intensity of the $572 \mathrm{keV}\left(17 / 2^{+}\right) \rightarrow\left(15 / 2^{+}\right)$transition is larger by about a factor of 2 in our data. A similar observation is made in our study of even-even Te $(Z=52)$ isotopes, populated in the same reaction, where the $6^{+} \rightarrow 4_{1}^{+}$transition is observed with about twice higher intensity compared to the spontaneous fission data [28]. The second main difference is the significantly stronger detection of the 928 and $1000 \mathrm{keV} \gamma$ rays, side-feeding the $\left(15 / 2^{+}\right)$state of the ground-state (g.s.) band from non-yrast states. Both observations are linked to the differences in analysis and trigger conditions while being relevant for the mechanisms of angular momentum generation in fission [40]. Likewise, the $500 \mathrm{keV}\left(19 / 2^{+}\right) \rightarrow\left(15 / 2^{+}\right)$transition in ${ }^{137} \mathrm{I}$ is about a factor 3 stronger in these data. It may be interesting to note that also several other intensities in ${ }^{137} \mathrm{I}$ are not consistent with the ${ }^{248} \mathrm{Cm}$ data, for example for the transitions connecting the $\left(29 / 2^{+}\right),\left(25 / 2^{+}\right),\left(21 / 2^{+}\right)$, and $\left(19 / 2^{+}\right)$ states. For the ${ }^{139} \mathrm{I}$ isotope the intensities are more comparable with the earlier data [14] with the exception of the intensities of the lowest-lying ground state transitions deexciting the $\left(11 / 2^{+}\right)$and $\left(9 / 2^{+}\right)$states. Here, the $\left(9 / 2^{+}\right) \rightarrow\left(7 / 2^{+}\right)$ transition is observed with the highest intensity, whereas the $\left(11 / 2^{+}\right) \rightarrow\left(9 / 2^{+}\right)$transition is the strongest one reported in Ref. [14].

Furthermore, when examining the intensity pattern in ${ }^{137} \mathrm{I}$, it has to be noted that some of the interband transitions are much stronger than those in the band. In particular, the $296 \mathrm{keV}\left(19 / 2^{+}\right) \rightarrow\left(17 / 2^{+}\right)$transition is rather strongly seen with $31(9) \%$ intensity, while the $\left(19 / 2^{+}\right)$state is fed only by $18(5) \%$ intensity, both relative to the strongest $553.8 \mathrm{keV}$ $\left(9 / 2^{+}\right) \rightarrow\left(7 / 2^{+}\right)$transition assumed as $100 \%$ intensity (see Table I). On the other hand, the $\left(15 / 2^{+}\right)$state is weakly fed $(<10 \%)$ and decays quite strongly to the lower-lying $\left(11 / 2^{+}\right)$ state. This might be an indicator for some unobserved sidefeeding. With the current level of statistics it is not possible to further investigate this possible feeding; however, it would be relevant for future studies of this nucleus.

\section{B. Remeasurement of the $\left(15 / 2^{+}\right)$state lifetime in ${ }^{135} I$}

The lifetime, $\tau$, of the $\left(15 / 2^{+}\right)$state was previously measured to be 2510(120) ps [20]. Being in the appropriate range for the $\mathrm{LaBr}_{3}(\mathrm{Ce})$-beam timing method, explained in Sec. II, it is used as a test case. Figure 5(a) shows the energy spectrum after applying a gate on the $\left(11 / 2^{+}\right) \rightarrow$ $7 / 2^{+}$transition in ${ }^{135} \mathrm{I}$. The spectrum is enlarged around the $288 \mathrm{keV}\left(15 / 2^{+}\right) \rightarrow\left(11 / 2^{+}\right)$transition which is visible in the spectra from both the HPGe and the $\mathrm{LaBr}_{3}(\mathrm{Ce})$ detectors. As the lifetime of the $\left(11 / 2^{+}\right)$state is much smaller than the one of the $\left(15 / 2^{+}\right)$state, both 1134 and $288 \mathrm{keV}$ time distribution can be added for the measurement of the $\left(15 / 2^{+}\right)$ state. This background-subtracted summed time distribution in the $\mathrm{LaBr}_{3}(\mathrm{Ce})$ detectors is shown in Fig. 5(b). According to the description in Sec. II, a fit value of $\tau=2340$ (115) ps is obtained. Note that we do not detect the $1696 \mathrm{keV}$ transition in the $\mathrm{LaBr}_{3}(\mathrm{Ce})$ detectors due to the narrow time window used for building the $\mathrm{LaBr}_{3}(\mathrm{Ce})$ events. Thus, the contribution of the higher-lying isomer (see Sec. III D) deduced from the intensities registered in the HPGe detectors is estimated to be below 27(6)\%. The fit presented in Fig. 5(b) extends only to about $50 \mathrm{~ns}$ to avoid contributions from time-delayed inelastic neutron scattering, that occur 10-15 ns after the prompt $\gamma$ rays. The shaded areas in Fig. 5(b) represent the uncertainty of the fit parameters and the variation of fit regions. Within the uncertainty the result is consistent with the previous measurement. In Ref. [20], the result was obtained as a weighted 

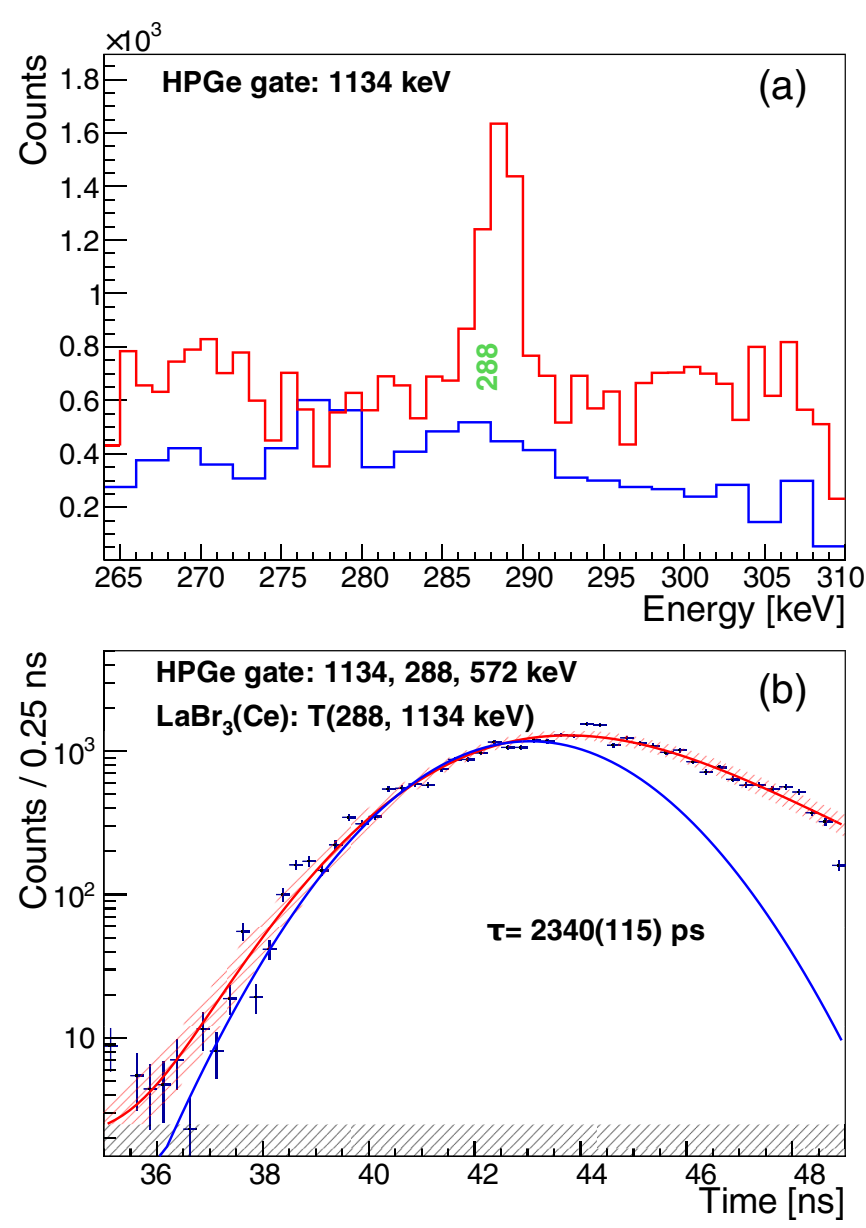

FIG. 5. Lifetime measurement of the $\left(15 / 2^{+}\right)$state in ${ }^{135} \mathrm{I}$. (a) HPGe (red) and $\mathrm{LaBr}_{3}(\mathrm{Ce})$ (blue) energy spectra gated on the $1134 \mathrm{keV}\left(11 / 2^{+}\right) \rightarrow 7 / 2^{+}$transition. (b) Background-subtracted $\mathrm{LaBr}_{3}(\mathrm{Ce})$ time distribution for the sum of the 288 and $1134 \mathrm{keV}$ transitions. The spectrum is fitted with a prompt Gaussian (blue) convoluted with an exponential decay curve plus a constant background, resulting in the full fit function (red).

average of four different values: $2610(260) \mathrm{ps}, 2670(200) \mathrm{ps}$, $2400(200)$ ps and 2380(200) ns. Our result agrees well with two of them and, as an independent measurement, reduces the relative uncertainty for the lifetime of this state.

\section{Lifetimes of the $\left(9 / 2^{+}\right)$states in ${ }^{137,139} \mathrm{I}$}

In the following, we demonstrate the lifetime measurements performed for the $\left(9 / 2^{+}\right)$states in the ${ }^{137,139} \mathrm{I}$ isotopes As explained in Sec. II, the $\mathrm{LaBr}_{3}(\mathrm{Ce})-\mathrm{LaBr}_{3}(\mathrm{Ce})$ time difference is applicable for sub-ns lifetimes as expected for these first-excited states. For the ${ }^{137}$ I nucleus, the lifetime of the $\left(9 / 2^{+}\right)$state can thus be obtained by measuring the time difference between the $554 \mathrm{keV}\left(9 / 2^{+}\right) \rightarrow\left(7 / 2^{+}\right)$and the $400 \mathrm{keV}\left(13 / 2^{+}\right) \rightarrow\left(9 / 2^{+}\right)$transition, i.e., $\Delta T(554,400)$. To tag the nucleus and the cascade of interest, a HPGe gate on 358 or $725 \mathrm{keV}$ above the $\left(13 / 2^{+}\right)$state is used. Afterwards, a $\mathrm{LaBr}_{3}(\mathrm{Ce})$ gate on the decaying $554 \mathrm{keV}$ transition is applied. Due to the low statistics, wide $\approx 20 \mathrm{keV} \mathrm{LaBr}{ }_{3}(\mathrm{Ce})$ gates were used. The spectra were investigated with the better energy resolution of the HPGe detectors for any kind of background or contaminants. Thus, the selection is chosen appropriately to contain clean $\gamma$ rays of interest.

The resulting energy projection is shown in Fig. 6(a) for $\mathrm{LaBr}_{3}(\mathrm{Ce})$ (blue) and HPGe (red) detectors. The $\mathrm{LaBr}_{3}(\mathrm{Ce})$ spectrum is generated from $\mathrm{HPGe}-\mathrm{LaBr}_{3}(\mathrm{Ce})-\mathrm{LaBr}_{3}(\mathrm{Ce})$ triple coincidences and the HPGe spectrum from HPGe$\mathrm{LaBr}_{3}(\mathrm{Ce})-\mathrm{HPGe}$ events. The $400 \mathrm{keV}$ feeding transition is clearly visible in both spectra [in Fig. 6(a)] as a result of the applied gates. A relatively high peak-to-background of around 3.0(3) is obtained for this peak. In a last step, a second $\mathrm{LaBr}_{3}(\mathrm{Ce})$ gate is applied on the feeding transition to generate the time difference spectrum shown in Fig. 6(c). The gate includes a background subtraction of regions around the peak of interest. Due to the low statistics, this may result in statistical fluctuations and over subtraction of individual bins; e.g., see Fig. 6(c). These fluctuations are considered by applying a full minimization procedure when determining the lifetime result. The time distribution is fitted with a convolution of a prompt Gaussian and an exponential decay plus a constant background, where the range of the prompt width is in accordance with the investigation of prompt transitions at these energies, discussed in Sec. II. The lifetime result of $\tau=390(70) \mathrm{ps}$ obtained in such analysis shows a typical example where the dominant error contribution comes from the variation of the prompt width rather than statistical fluctuations.

To measure the lifetime of the $\left(9 / 2^{+}\right)$state in the ${ }^{139} \mathrm{I}$ isotope, the time difference between the $419 \mathrm{keV}\left(9 / 2^{+}\right) \rightarrow$ $\left(7 / 2^{+}\right)$decaying transition and the $397 \mathrm{keV}\left(13 / 2^{+}\right) \rightarrow$ $\left(9 / 2^{+}\right)$feeding transition is considered. To tag the nucleus of interest, ${ }^{139} \mathrm{I}$, a HPGe gate on the $464 \mathrm{keV}\left(17 / 2^{+}\right) \rightarrow$ $\left(13 / 2^{+}\right)$transition is used. Then, a $\mathrm{LaBr}_{3}(\mathrm{Ce})$ gate on $397 \mathrm{keV}$ is applied, resulting in the spectra of Fig. 6(b). Despite the higher background level as compared to the ${ }^{137} \mathrm{I}$ case and the reduced statistics due to the lower population of ${ }^{139} \mathrm{I}$, a clear identification of the $419 \mathrm{keV}$ transition decaying out the $\left(9 / 2^{+}\right)$state is possible. With a peak-to-background ratio of 1.7(3) it is about a factor of 2 lower than for the less exotic ${ }^{137} \mathrm{I}$. The time difference spectrum after applying the additional $419 \mathrm{keV}$ gate is presented in Fig. 6(d). The different slope on the right side of the distribution when compared to ${ }^{137} \mathrm{I}$ already indicates a shorter lifetime of the state in ${ }^{139} \mathrm{I}$. This is confirmed by the result of the applied convolution fit, emerging in a value of $\tau=130(50)$ ps. As expected because of the lower population, the statistical uncertainty is larger compared to ${ }^{137} \mathrm{I}$ and dominates the lifetime result in ${ }^{139} \mathrm{I}$. The results are listed in Table II.

\section{Summary of lifetime results}

In addition to the $\left(9 / 2^{+}\right)$states, the lifetimes of the $\left(13 / 2^{+}\right)$ and $\left(17 / 2^{+}\right)$states in the ${ }^{137,139} \mathrm{I}$ isotopes are measured in the same way as described in Sec. III C. Time difference spectra are shown in Fig. 7. A particular case is the lifetime of the $\left(13 / 2^{+}\right)$state in ${ }^{137}$ I. It has a strong feeding transition of $358 \mathrm{keV}$ and two decaying transitions of 400 and $334 \mathrm{keV}$ that are both reasonably strong (with relative intensities of $I_{\gamma}=82 \%$ and $15 \%$, respectively). This provides the possibility to measure two lifetimes independently from 

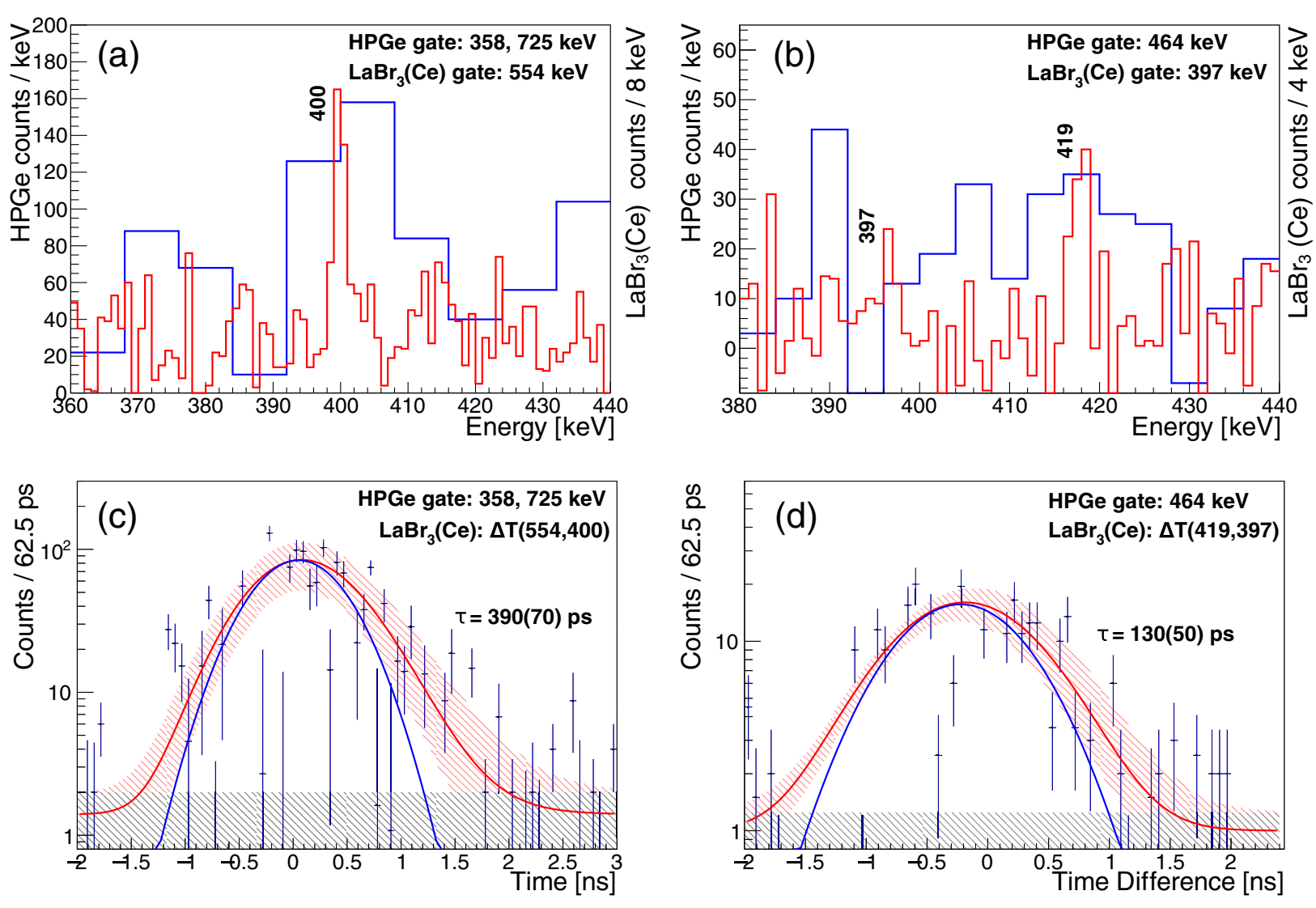

FIG. 6. HPGe (red) and $\mathrm{LaBr}_{3}(\mathrm{Ce})$ (blue) energy spectra after a HPGe sum or single gates and an additional $\mathrm{LaBr}(\mathrm{Ce})$ gate for transitions in (a) ${ }^{137} \mathrm{I}$ and (b) ${ }^{139} \mathrm{I}$ isotopes. The respective gates are labeled. Time difference spectra for the $\left(9 / 2^{+}\right)$state in (c) ${ }^{137} \mathrm{I}$ and (d) ${ }^{139} \mathrm{I}$ nuclei. The lifetimes result from a convolution fit (red) of a prompt Gaussian (blue) and an exponential decay plus constant background. The shaded area indicate the uncertainty bands from variation of parameters and fit regions.

the same data. We note that while the $400 \mathrm{keV}$ transition is of $E 2$ type, the $334 \mathrm{keV}$ transition is of $(M 1+E 2)$ type with a small $E 2$ mixing $(\delta=+0.08)$ as taken from Ref. [24]. The $\Delta T(400,358)$ and the $\Delta T(334,358)$ time distributions are depicted in Figs. 7(a) and 7(b), respectively. From the fit of the $\Delta T(400,358)$ distribution, a lifetime of $140_{-50}^{+40} \mathrm{ps}$ could be obtained. The resulting lifetime of the $\Delta T(334,358)$ distribution amounts to $145(60) \mathrm{ps}$, reflected by the larger uncertainty due to lower statistics, while at the same time being consistent with the previous lifetime result. Because of the smaller uncertainty, the lifetime $\tau=140_{-50}^{+40} \mathrm{ps}$ may be adopted. However, we list both results and their respective transition rates in Table II. We also note that this is the only case where both (decaying) transitions are given separately for comparison, while for the other states listed in the table the transition rates represent the lifetime result of the strongest transition.

For the $\left(17 / 2^{+}\right)$state in ${ }^{137} \mathrm{I}$, the lifetime is measured via the $\Delta T(358,725)$ time difference. The time distribution is shown in Fig. 7(c) and, from the fit, a lifetime of 150(50) ps is obtained (see Table II). Despite the lower population of this state, compared to the $\left(13 / 2^{+}\right)$state, the uncertainty in the result is better due to lower statistical fluctuations at the measured energies.
For the ${ }^{139}$ I nucleus, the measured time differences for the $\left(13 / 2^{+}\right)$and the $\left(17 / 2^{+}\right)$states are the $\Delta T(397,464)$ and the $\Delta T(464,481)$ spectra, respectively. Figure $7(\mathrm{~d})$ shows the time distribution used for the lifetime determination of the $\left(13 / 2^{+}\right)$state. From the fits, a lifetime value of $120(60)$ ps is deduced and can be found in the last section of Table II. In the case of the $\left(17 / 2^{+}\right)$state in the ${ }^{139}$ I isotope, only an upper limit of $70 \mathrm{ps}$ can be given from the analysis. The reduced statistics in the lifetime determination is caused by narrow gates due to close-lying populating and depopulating transitions.

The lifetimes of the $\left(11 / 2^{+}\right)$states in all ${ }^{135-139}$ I isotopes are too short to be measured with the convolution method. Thus, upper limits could only be determined as given in Table II. In the case of the ${ }^{135} \mathrm{I}$ isotope, the fact that the $\left(11 / 2^{+}\right)$state is much shorter than the $\left(15 / 2^{+}\right)$state is consistent with the previous observation [20]. These lifetime limits are additionally cross checked for ${ }^{135,137}$ I by analyzing the centroid difference of delayed and antidelayed time distributions. For details on the centroid difference method the reader is referred to Ref. [27].

An examination of the $\left(23 / 2_{1}^{-}\right)$and $\left(29 / 2_{1}^{+}\right)$states in respectively the ${ }^{135}$ I and ${ }^{137}$ I nuclei is also performed according to the expectations of the previous works (see Sec. IV). For 
TABLE II. Summary of the lifetime measurements in neutron rich iodine isotopes. Reduced transition probabilities are corrected for internal conversion assuming a nonmixed $M 1$ or $E 2$ transition using [42], unless explicitly noted otherwise. $B(E 2)$ transitions strengths are given in $e^{2} \mathrm{fm}^{4}$ and $B(M 1)$ values in $\mu_{N}^{2}$. For mixed $(M 1+E 2)$ transitions, $B(M 1)$ values are provided. Shell-model values are calculated in the $r 4 h$ - $r 5 i$ model space ranging from $Z=50, N=82$ to $Z=82, N=126$ using the N3LOP effective interaction (SM-I) and Kuo-Herling (SM-II) interaction with set of effective charges $\left(e_{\pi}, e_{v}\right)$ of $(1.6 e, 0.6 e)$ for SM-I, SM-IIb, $(1.5 e, 0.5 e)$ for SM-IIa, $0.7 g_{\text {free }}$ for SM-I, SM-IIb, and $0.6 g_{\text {free }}$ for SM-IIa. For further details see text.

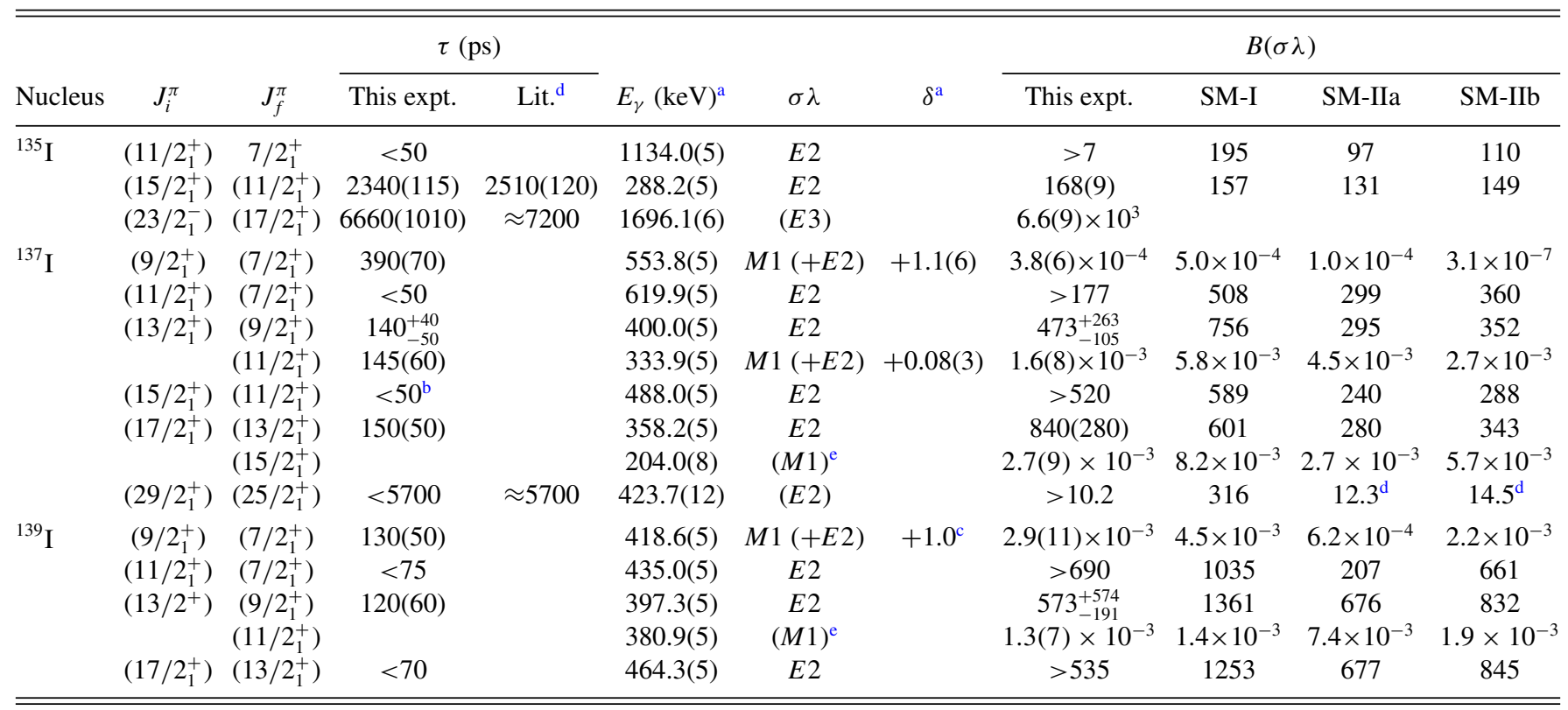

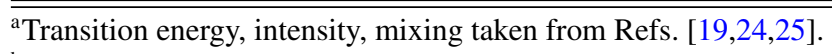

${ }^{\mathrm{b}}$ From effective lifetime.

${ }^{\mathrm{c}} B(M 1)$ value calculated using $\delta$ from Ref. [25] $(\alpha(M 1)=0.0151$ [14,25]). The limits for pure $M 1$ or $E 2$ transitions correspond to $B(M 1)=5.8(22) \times 10^{-3} \mu_{N}^{2}$ and $B(E 2)=482(186) e^{2} \mathrm{fm}^{4}$, respectively.

${ }^{\mathrm{d}}$ For literature values see text.

${ }^{\mathrm{e}} B(M 1)$ values given as limits assuming a pure $M 1$ transition.

the $3689.8 \mathrm{keV}\left(23 / 2_{1}^{-}\right)$state in the ${ }^{135}$ I isotope, based on the $1696 \mathrm{keV}$ transition detected in the HPGe detectors, a value of 6660(1010) ps $\left[T_{1 / 2}=4.6(7) \mathrm{ns}\right]$ could be obtained, while this transition is not observed in the $\mathrm{LaBr}_{3}(\mathrm{Ce})$ detectors. The result is well in agreement with the earlier existing information, e.g., a lifetime less than $7.2 \mathrm{~ns}$ ( $T_{1 / 2}$ of $\left.5 \mathrm{~ns}\right)$ is quoted in Ref. [43], much shorter than a previous estimate (Ref. [13] of [43]). An upper bound without an accurate value of $5 \mathrm{~ns}$ $\left(T_{1 / 2}<3.5 \mathrm{~ns}\right)$ for the lifetime is also given in Ref. [44]. All results are summarized in Table II, together with the final outcomes for the reduced transition rates.

This work provides the first lifetime measurements in the neutron-rich ${ }^{137,139}$ I isotopes. From these lifetimes, first results on the reduced transition probabilities could be established. In the cases where $\Delta J=J_{f}-J_{i}=1$ and the mixing ratio is known, the mixed $B(M 1)$ strength is given. For the $\left(17 / 2^{+}\right) \rightarrow\left(15 / 2^{+}\right)$transition in ${ }^{137} \mathrm{I}$, no mixing ratio is known and the mixed $B(M 1)$ value can be taken as an up-
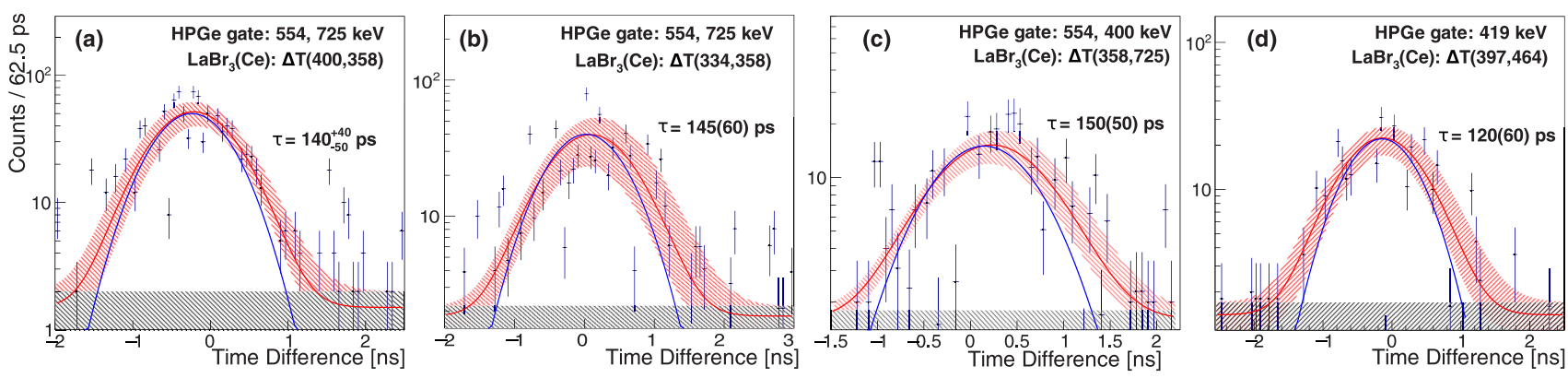

FIG. 7. Time difference spectra for the measured (a), (b) $\left(13 / 2^{+}\right)$and (c) $\left(17 / 2^{+}\right)$states in ${ }^{137}$ I, and (d) the $\left(13 / 2^{+}\right)$state in ${ }^{139}$ I. Respective HPGe gates (to select the cascade of interest) and the $\operatorname{LaBr}_{3}(\mathrm{Ce})$ time differences $(\Delta T)$ are labeled. Each spectrum is fitted with a convolution of a prompt Gaussian (blue) and an exponential decay plus constant background (black). Shaded areas represent parameter fluctuations in the fits. 
per limit, given the intensity ratio of the observed decaying transitions. For the $B\left(M 1 ;\left(9 / 2^{+}\right) \rightarrow\left(7 / 2^{+}\right)\right)$value in the ${ }^{139} \mathrm{I}$, a mean mixing ratio of $\delta=1.0$ is assumed, based on the conversion coefficient $\alpha=0.0151$ (9) from Refs. [14,25]. To estimate the uncertainty in this transition rate, limits assuming an unmixed transition $\alpha(M 1)=0.0159$ are used, while a pure $E 2$ transition $[\alpha(E 2)=0.0143]$ would result in $B(E 2)$ of $482(186) e^{2} \mathrm{fm}^{4}[25,42]$. The $381 \mathrm{keV}\left(13 / 2^{+}\right) \rightarrow$ $\left(11 / 2^{+}\right)$transition is consistent with an $M 1$ multipolarity, obtained in angular correlation and polarization measurements [14]. In this case, a similar $\delta$ is assumed for such mixed $B\left(M 1 ;\left(13 / 2^{+}\right) \rightarrow\left(11 / 2^{+}\right)\right)$strength and can also be taken as a lower experimental limit. To deduce the $B(E 2)$ value of the $\left(17 / 2^{+}\right) \rightarrow\left(13 / 2^{+}\right)$transition in the ${ }^{139}$ I nucleus, the nonobservation of the previously reported $351 \mathrm{keV}\left(17 / 2^{+}\right) \rightarrow$ $\left(15 / 2^{+}\right) \gamma$ ray (see Ref. [25]) is considered. Therefore, the $B\left(E 2 ; 17 / 2^{+} \rightarrow 13 / 2^{+}\right)$transition rate assumes only one decay branch out of the $\left(17 / 2^{+}\right)$state and may be regarded as an effective value lower limit. For the $B\left(E 3 ;\left(23 / 2^{-}\right) \rightarrow\right.$ $\left.\left(17 / 2^{+}\right)\right)$transition rate, a pure $(E 3)$ transition is examined, as identified in Refs. [41,43,44], providing the first such result with a reasonable significance.

\section{SHELL-MODEL CALCULATIONS AND DISCUSSION}

The experimental results are compared to theoretical predictions from large-scale shell-model calculations. The $r 4 h-r 5 i$ model space above a ${ }^{132} \mathrm{Sn}$ core is used, consisting of the $1 f_{7 / 2}, 0 h_{9 / 2}, 1 f_{5 / 2}, 2 p_{3 / 2}, 2 p_{1 / 2}, 0 i_{13 / 2}$ orbitals for neutrons and $0 g_{7 / 2}, 1 d_{5 / 2}, 1 d_{3 / 2}, 2 s_{1 / 2}, 0 h_{11 / 2}$ for protons. The matrix elements are derived from an N3LOP effective interaction. Single-particle energies for neutrons and protons are taken from experimental data on ${ }^{133} \mathrm{Sn}$ and ${ }^{133} \mathrm{Sb}$ [45]. The $0 i_{13 / 2}$ neutron and $2 s_{1 / 2}$ proton orbital energies are taken from Refs. [46,47], respectively. The N3LOP effective interaction was successfully used before in describing the spectroscopic properties and collectivity of nuclei in the vicinity of the ${ }^{132} \mathrm{Sn}$ nucleus $[5,7,8,15,28,48]$. The diagonalization of the considered systems ${ }^{135,137,139} \mathrm{I}$ is achieved using the ANTOINE shell-model code $[49,50]$. These calculations are further denoted as SM-I. The results are illustrated in Table II and are discussed in the following sections. In addition, for comparison, we performed standard shell-model calculations using the NUSHELLX code [51] and the $(N>82, Z>50)$ Kuo-Herling interaction $(\mathrm{KH})$ [52,53] already used in this region. The single-particle energies are taken from experimental spectra of $A=207$ nuclei [53] with a single hole to the doubly magic ${ }^{208} \mathrm{~Pb}$ nucleus. The two-body matrix elements are modified according to fits on level energies of ${ }^{206} \mathrm{~Pb},{ }^{206} \mathrm{Tl}$, and ${ }^{206} \mathrm{Hg}$ isotopes below ${ }^{208} \mathrm{~Pb}$ [54]. The interaction is widely used to successfully describe experimental data on nuclei "southeast" of ${ }^{208} \mathrm{~Pb}$ (e.g., Refs. [55-57]) as well as data on $N=84$ isotones [58] and neutron-rich $\mathrm{Sb}$ isotopes [59-61].

The two shell-model (SM) calculations achieved using the N3LOP and KH interactions are further denoted as SM-I and SM-II, respectively. The $B(E 2)$ transitions are calculated using the proton-neutron effective charges, which are $\left(e_{\pi}, e_{\nu}\right)$ of $(1.6 e, 0.6 e)$ (SM-I and SM-IIb) and (1.5e, 0.5e) (SM-IIa). For the $B(M 1)$ transitions the SM-I calculations are performed using quenched spin and orbital $g$ factors [5], a quenched $g$ factor of $0.7 g_{\text {free }}$ for SM-IIb [4] and $0.6 g_{\text {free }}$ for SM-IIa.

\section{A. Excitation energies and configurations}

\section{Intermediate spin states}

The three valence-proton nucleus ${ }_{53}^{135} \mathrm{I}_{82}$ exhibits features of both single-particle and collective excitations despite being semimagic. When contrasting it to the neighboring $N=84,86$ isotopes, their level sequence is strongly compressed, as it would be expected with the development of collectivity. In the level scheme of ${ }^{135} \mathrm{I}$, transitions involving the $\left(9 / 2^{+}\right)$state, known at $1184 \mathrm{keV}$ [19], are not observed neither in this work nor in previous fission studies $[41,43,62]$. This state becomes the first excited one detected in the ${ }^{137,139} \mathrm{I}$ isotopes (see Fig. 3). As can be seen for these nuclei, the decay out of the $\left(9 / 2^{+}\right)$state is the strongest, while the previously known $\left(5 / 2^{+}\right)$state, believed to be the first excited one and seen in the previous fission works, is also not detected here. In ${ }^{135} \mathrm{I}$, further differences can be found with respect to the heavier isotopes, such as the lack of separate branches with interconnecting transitions, while such interband transitions become typical when moving away from the $N=82$ closedneutron shell. In Fig. 3, these can especially be recognized in the ${ }^{137}$ I nucleus, while appearing weaker in the ${ }^{139} \mathrm{I}$ isotope, where the levels are even further compressed.

The yrast positive-parity states up to $J=15 / 2$ are built on the $\pi\left(0 g_{7 / 2}^{3}\right)$ configurations where the maximum spin can be achieved in a fully aligned coupling, as confirmed by the shell-model calculations. The $\left(17 / 2^{+}\right)$state in the ${ }^{137} \mathrm{I}$ isotope is based on a $\pi\left(0 g_{7 / 2}^{2} 1 d_{5 / 2}^{1}\right)$ configuration involving the excitation from the $\pi\left(0 g_{7 / 2}\right)$ to the $\pi\left(1 d_{5 / 2}\right)$ orbital. This configuration was proposed also for the ${ }^{135} \mathrm{I}$ nucleus $[41,43]$, in a full agreement with our shell-model calculations (SM-I/II; see Sec. IV A 2).

\section{Specific positive-parity states}

Several low-lying yrast states are examined in more detail in this work. Their evolution as a function of the mass number is compared to the theoretical results from SM-I and SM-II in Figs. 8 and 10. For the lowest states, a very good agreement for SM-I and a reasonable agreement for SM-II between the experimental levels and the shell-model predictions can be observed. The overall tendency and level sequences is relatively well reproduced by the theory with tolerable differences of the order of 50 to $200 \mathrm{keV}$ with SM-I for the $\left(15 / 2^{+}\right)$and $\left(17 / 2^{+}\right)$ states and larger for SM-II.

The drop in excitation energies for all states, as discussed above, can be well followed when moving away from the $N=82$ closed shell in ${ }^{135}$ I towards ${ }^{137} \mathrm{I}$, while this is somewhat reduced (flat line) between $N=84$ and $N=86$ for the ${ }^{139} \mathrm{I}$ isotope. The effect is strongest for the $\left(5 / 2^{+}\right)$and $\left(17 / 2^{+}\right)$ states to which we can add the behavior of the $\left(13 / 2^{+}\right)$states due to their common origin. The evolution of these states can be traced in Fig. 8. According to our shell-model (SM-I) results, the set of states have the pure (in $A=135$, e.g., $\approx 90 \%$ ) to dominant (in $A=137,139$, e.g., 23-15\%) proportion based on the $\pi\left(g_{7 / 2}^{3}\right)$ configuration. Similarly, the $7 / 2^{+}$g.s. and the 


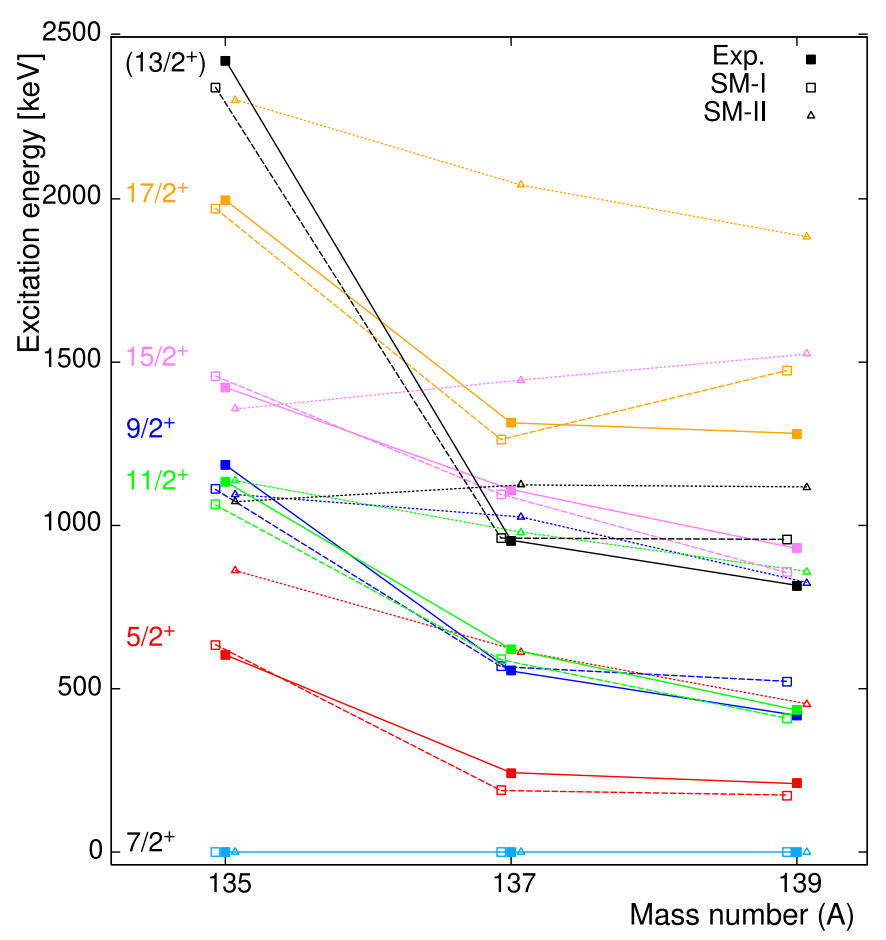

FIG. 8. Energy of excited states as a function of mass number for ${ }^{135,137,139}$ I. Experimental values are taken from the evaluated nuclear data sheets $[19,24,25]$ and are shown with filled squares. The identification of $\left(13 / 2^{+}\right)$state in $A=135$ is tentative. Theoretical values from the present shell-model calculations are shown with empty squares (SM-I) and empty triangles (SM-II). Solid and dashed lines are drawn to guide the eye. Each sequence is labeled with spin and parity.

$\left(11 / 2^{+}\right)$and $\left(15 / 2^{+}\right)$states are coupled to $v\left(f_{7 / 2}^{v}\right), v=2,4$ neutrons, respectively. Interestingly, they belong to a different sequence in the decay schemes and this is clearly visible in their level schemes (Fig. 3) with evident band structure for the ${ }^{137}$ I and ${ }^{139}$ I nuclei. According to SM-I, these $\left(9 / 2^{+} 13 / 2^{+}\right.$, $\left.17 / 2^{+}\right)$states originate from the same proton excitation to the $\pi\left(d_{5 / 2}\right)$ orbital with dominant $\pi\left(g_{7 / 2}^{2} d_{5 / 2}\right)$ configuration coupled as for the other states (in $A=137,139$ ) to the even neutrons in the $v\left(f_{7 / 2}\right)$ orbit. As discussed above for $A=135$, only the $\left(17 / 2^{+}\right)$state originates from such an excitation, which reflects its relatively large energetic difference (thus orbital positioning) with respect to the $v\left(g_{7 / 2}^{3}\right)$-based $7 / 2^{+}$g.s. in this nucleus. It should be noted that, while the $\left(9 / 2^{+}\right)$state changes its origin between $N=82$ and $N=84$ (not even populated in the yrast sequence of $\left.{ }^{135} \mathrm{I}\right)$, the $\left(17 / 2^{+}\right)$state stays a relatively pure member of this configuration (e.g., according to SM-I about $50 \%$ in $A=137$, and in $A=139$ it is the maximal partition with about $20 \%$ ). Thus, its more detailed investigation (e.g., with the transition strength of its deexcitation) is interesting. The $\left(13 / 2^{+}\right)$state, on the other hand, has a more mixed origin. According to the calculations, some neutron components with excitations to the $v\left(p_{3 / 2}\right)$ orbital with $\pi\left(g_{7 / 2}^{2} d_{5 / 2}\right) v\left(f_{7 / 2}^{3} p_{3 / 2}\right)$ configuration are expected to occur. This is visible also in the orbital occupations for the three nuclei of interest, which we show in Fig. 9. In this figure, in addition to SM-I we added the predictions of SM-II, thus comparing two shell-model interactions (see the beginning of Sec. IV). Agreement on the origin for some of the states, while disagreement for others, partially the subject in the previous works on ${ }^{135-139}$ I nuclei $[14,22,41,43]$, can be seen.

The $N=82$ case may be considered simpler as the lowlying states have practically no neutron component; e.g., as discussed above, the neutron excitations from the closed $\mathrm{Sn}$ core take place at excitation energy above $4 \mathrm{MeV}$. However, the orbital contributions are different, especially for the $\pi\left(d_{5 / 2}\right)$ orbital that apparently is important for low-lying states in $A=135$ in the SM-I while it is not the case in SM-II. This could be explained by the overestimated $\pi\left(g_{7 / 2}\right)-\pi\left(d_{5 / 2}\right)$ proton gap in the $\mathrm{KH}$ case compared to the N3LOP case (see Fig. 8). The relatively shared occupation in these $\pi\left(d_{5 / 2}\right)$ and $\pi\left(g_{7 / 2}\right)$ proton orbitals is visible for SM-I (N3LOP) with the mass increase up to $A=139$, where they are practically competing (see Fig. 9). This reflects their close positioning at $N=86$. This is also in disagreement with the excitedstates origin by SM-II, predicting the occupation, respectively, closer positioning of the $\pi\left(d_{3 / 2}\right)$ orbital in $N=84$ and the $\pi\left(s_{1 / 2}\right)$ and $\pi\left(d_{5 / 2}\right)$ orbitals at $N=86$. On the neutron side, $v\left(f_{7 / 2}\right)$ is the main component in all wave functions with some small influence of the $v\left(p_{3 / 2}\right)$ orbital as noted above, reflecting their proximity. This occupation increases very slightly according to SM-I between $N=84$ and $N=86$, which would reflect the decreasing distance between these orbitals, with no drastic effects with the increase of $N$ as elaborated in some of our earlier works $[7,15]$. For the neutrons, SM-II also predicts strong fractioning to the $v\left(h_{9 / 2}\right)$ and $v\left(i_{13 / 2}\right)$ orbitals, in contrast with SM-I where these partitions are much smaller for the examined states and become important only at much higher energy. Note that in the two calculations the excitations are treated as valence particles (SM-I) and valence holes (SM-II). Further discussion on the origin will be given in the context of transition rates inferred from the measured timing properties of these states (see Sec. IV C).

\section{Negative-parity states}

In this work we observe two negative-parity $\left(19 / 2^{-}\right)$and $\left(23 / 2^{-}\right)$states with their high-energy (i.e., $E_{\gamma}>1.6 \mathrm{MeV}$ ) connections with the yrast states. As can be seen in Fig. 3, the population of these states makes the level scheme of ${ }^{135}$ I particularly different as compared to the ${ }^{137,139}$ I isotopes. Identified in Ref. [43] based on shell-model calculations, it was suggested that their origin involves the proton excitations to the $\pi\left(0 h_{11 / 2}\right)$ shell, dominated by a $\pi\left(0 g_{7 / 2}^{2} 0 h_{11 / 2}^{1}\right)$ component in the wave functions. As discussed above (and in Ref. [43]), except with the $\mathrm{KH}$ effective interaction, this suggestion is in good agreement also with the results using the N3LOP effective interaction; see Sec. IV. One may note here that except for the description of excitations in ${ }^{135} \mathrm{I}$ by a simple single-proton (in $g_{7 / 2}$ ) coupling to states in the ${ }^{134} \mathrm{Te}$ nucleus $[43,47]$, the transition rate $B\left(E 3 ;\left(23 / 2^{-}\right) \rightarrow\left(17 / 2^{+}\right)\right)$ of $8.7 \times 10^{3} e^{2} \mathrm{fm}^{6}$ was estimated in Ref. [44]. It was based on pure $\pi\left(g_{7 / 2}^{2} \otimes h_{11 / 2}\right)_{23 / 2-}$ and $\pi\left(g_{7 / 2}^{2} \otimes d_{5 / 2}\right)_{17 / 2+}$ configurations, corresponding to a transition between $\pi h_{11 / 2}$ and $\pi d_{5 / 2}$ proton orbits. This is in good agreement with our ex- 


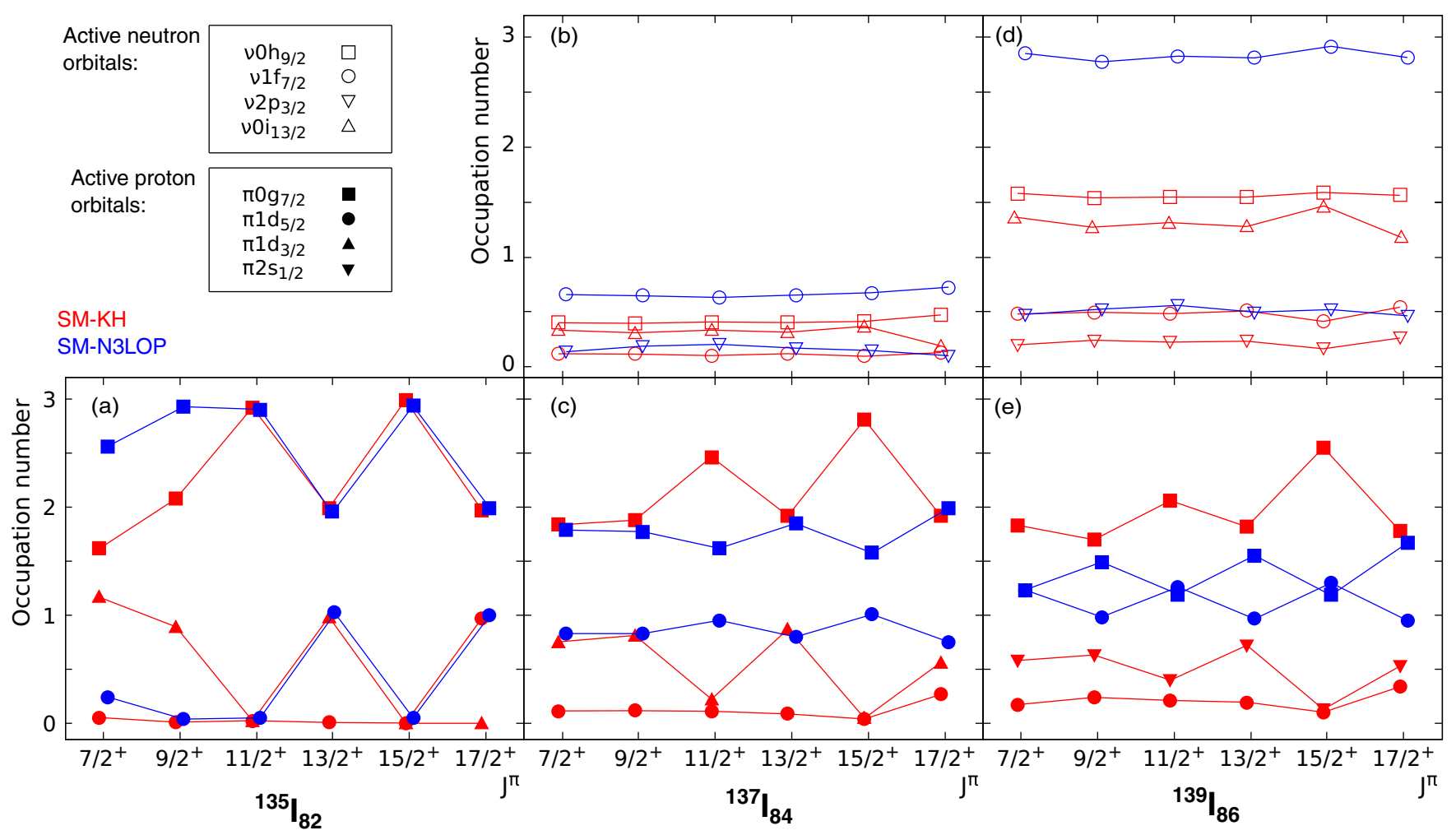

FIG. 9. Occupations for the most prominent proton or neutron-proton configurations, respectively, in the ${ }^{135} \mathrm{I}$ (a), the ${ }^{137} \mathrm{I}$ (b,c), and the ${ }^{139} \mathrm{I}$ (d), (e) isotopes as calculated by the shell-model using the N3LOP and the Kuo-Herling (KH) shell-model interactions. See text for details.

perimental result [6.6(9) $10^{3} e^{2} \mathrm{fm}^{6}$; see Table II] inferred from the measured lifetime of the $\left(23 / 2^{-}\right)$state. Therefore, it provides interesting qualitative information on the collective enhancement of the $E 3$ transition rate at this semimagic nucleus which is not available for the heavier-mass isotopes. Note that such negative parity states are not observed for the ${ }^{137,139}$ I isotopes, either in this work or in the previous works evaluated in Refs. [24,25].

\section{Other non-yrast states}

Another particularity in the ${ }^{135} \mathrm{I}$ isotope, observed here, is the detection of 928 and $1000 \mathrm{keV} \gamma$ feeding branches to the $\left(15 / 2^{+}\right)$state with $E_{x}=2350.6 \mathrm{keV}$ and $E_{x}=2421.8 \mathrm{keV}$, respectively. Deexciting possibly non-yrast states, these transitions were detected previously [19] though no spin-parity assignment could be proposed. For example, these states are particularly distinct from the positive-parity $\left(15 / 2^{+}\right)$and $\left(17 / 2^{+}\right)$states in the level scheme of ${ }^{135} \mathrm{I}$, and at the same time are well separated in energy from the other known negative-parity states such as $\left(19 / 2^{-}\right)$and $\left(23 / 2^{-}\right)$discussed above. According to our shell-model calculations, candidates that would be in conjunction with their energies, intensities, and decay would be $\left(11 / 2^{-}\right)$and $\left(13 / 2_{2}^{+}\right)$states, predicted at 2225 and $2339 \mathrm{keV}$ of excitation energy, respectively. Note that other negative-parity states either have larger $\Delta J$ or are far in energy, e.g., the rest of the positive parity states are predicted to be higher and with different origin (see Fig. 10). Taking into account that the non-negligible $M 1$ strength between a potentially populated $\left(15 / 2_{2}^{+}\right)$and $\left(13 / 2_{2}^{+}\right)$or an
$E 2$ between $\left(15 / 2_{2}^{+}\right)$and $\left(11 / 2_{2}^{+}\right)$is calculated, these states cannot be excluded. It is relevant to note that all the positiveparity candidates have almost pure $\pi\left(g_{7 / 2}^{2} d_{5 / 2}\right)$ configuration (e.g., $\geqslant 95 \%$ according to SM) which would correspond to an enhanced branching to the $\left(15 / 2^{+}\right)$state with $\pi\left(g_{7 / 2}^{3}\right)$ configuration $(\approx 96 \%$ according to $S M)$. Therefore, this corresponds to a transition between $\pi\left(d_{5 / 2}\right)$ and $\pi\left(g_{7 / 2}\right)$ orbitals, taking into account the high purity in the calculated states. They will thus be similar (and in the same energy range) as the transitions between the $\left(17 / 2^{+}\right)\left(\pi g_{7 / 2}^{2} d_{5 / 2}\right)$ and the $\left(15 / 2^{+}\right)$ $\left(\pi g_{7 / 2}^{3}\right)$ states, identified in Refs. [41,43]. On the other hand, the negative-parity $\left(11 / 2^{-}\right)$candidate has pure $\pi\left(g_{7 / 2}^{2} h_{11 / 2}\right)$ $(>85 \%)$ configuration as all negative-parity states and would be its populated member with lowest energy. Thus, a branch between an $\left(11 / 2^{-}\right)$and the known $\left(15 / 2^{+}\right)$state would rather correspond to a $\pi\left(h_{11 / 2}\right) \rightarrow \pi\left(g_{7 / 2}\right)$ transition and become the first identified connection of the same type as with the $\left(17 / 2^{+}\right)$ states though this time with the $\left(15 / 2^{+}\right)$state sitting $572 \mathrm{keV}$ below. Note that the type of transition between $\pi\left(h_{11 / 2}\right)$ and $\pi\left(d_{5 / 2}\right)$ was suggested in Refs. [41,43] to connect the higherlying negative parity states with the $\left(17 / 2^{+}\right)$state, identified as the lowest-energy member of the $\pi\left(g_{7 / 2}^{2} d_{5 / 2}\right)$ multiplet in ${ }^{135} \mathrm{I}$.

\section{B. Links to the ${ }_{52} \mathrm{Te}$ and ${ }_{55} \mathrm{Cs}$ isotones}

In the level schemes of the nuclei of interest to this work some particular relations to other isotones are present. In the case of the ${ }^{135}$ I nucleus, other than those previously discussed 

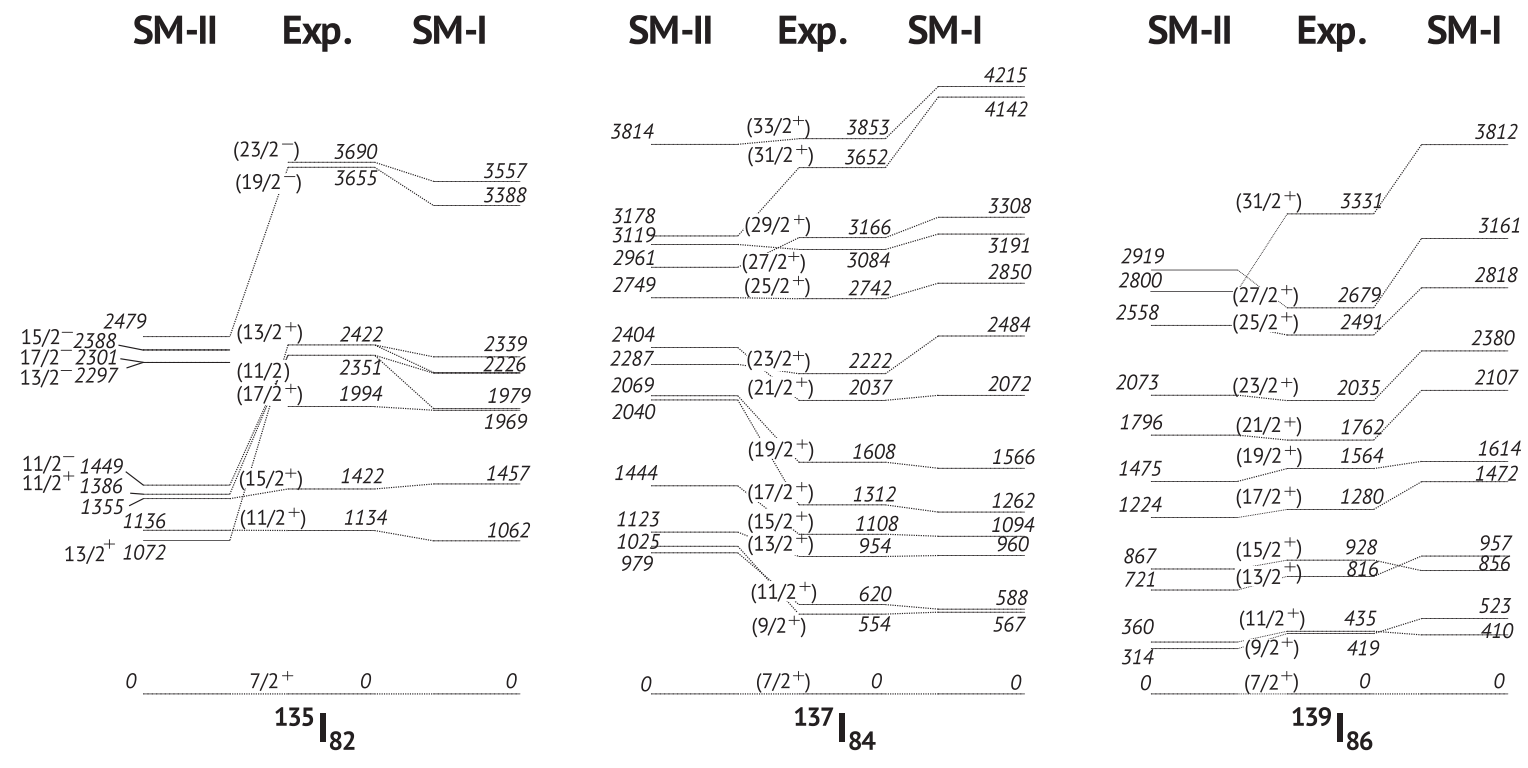

FIG. 10. Comparison of experimental and theoretical (SM-I and SM-II) excitation energies for the ${ }^{135,137,139}$ I isotopes. See text for details.

(see Sec. IV A), higher-energy feeders were previously reported in Ref. [41]. These $\gamma$-ray transitions with energy of the order of $2 \mathrm{MeV}$ connecting with the $\left(15 / 2^{+}\right)$state are unobserved here. Known from Refs. [41,43] to deexcite the higher-lying core-excited states (with $E_{x}>4 \mathrm{MeV}$ ), these are the states representing the $\pi\left(g_{7 / 2}^{3}\right) \nu\left(f_{7 / 2}^{1} h_{11 / 2}^{-1}\right)$ configuration. As discussed above, this relates particularly well the behavior of ${ }^{135} \mathrm{I}$ with the semimagic neighbor ${ }^{134} \mathrm{Te}$, as the large shell gap is present for both two-proton and three-proton isotones of the doubly magic ${ }_{50}^{132} \mathrm{Sn}$. Very weakly, in this work we observe only one member of this multiplet, the $\left(19 / 2^{+}\right)$state, at an excitation energy of about $4.2 \mathrm{MeV}$. Being the lowest member of this broken neutron-core excitation, it appears almost at the same energy as in ${ }^{134} \mathrm{Te}$ [43].

In Ref. [22], it was expected that the extra proton collectivity would cause significantly close-lying levels and due to low-energy transition imply a long-lived isomer at high excitation energy. The nonobservation of such a $12^{+}$isomeric state in ${ }^{136} \mathrm{Te}$ triggered a corresponding search in the ${ }^{137} \mathrm{I}$ nucleus [23] for a state of spin-parity $\left(29 / 2^{+}\right)$. In Ref. [22], it was concluded that the limited model space of the shell-model calculations with the Kuo-Herling interaction and an empirical set of single-particle energies is not appropriate to account for the data, and further data are needed to search for the real effect of the protons. The authors of the subsequent work [23] agreed that the model space limitation resulted in insufficient performance for the excitations in ${ }^{137} \mathrm{I}$. This was attributed to the isomer in ${ }^{137}$ I while performing a different shell-model calculation. Their expectations for the $\left(29 / 2^{+}\right)$state are based on the $\pi\left(g_{7 / 2}^{2} d_{5 / 2}\right) v\left(f_{7 / 2}^{2}\right)$ configuration, in good agreement with our shell-model results with $>86 \%$ of this strong and high-lying in energy dominating $\pi\left(d_{5 / 2}\right)$ excitation. Neither of the works in Refs. [22,23] observed such an isomer, nor can we find one in our work, where an upper limit for the $T_{1 / 2}$ of the order of $4 \mathrm{~ns}$ can be set. For an assumed $E 2$ decay to the $\left(25 / 2^{+}\right)$state that is the only observed decay branch, our result would be in agreement with a factor of about 100 hindrance, suggested in [23].

In Ref. [43], 11/2 $2_{1}^{+}$(and other yrast) states in the ${ }_{55}^{133-141} \mathrm{Cs}$ isotopes were described as a simple coupling of the valence proton to the even ${ }_{54} \mathrm{Xe}$ core. This was examined up to $N=86$ for the isotone of our ${ }^{139} \mathrm{I}$ nucleus of interest. As already stated, similar behavior was found in the case of ${ }_{53} \mathrm{I}$ where states could be described by couplings to the ${ }_{52} \mathrm{Te}$ core. The identification of corresponding states in ${ }^{137} \mathrm{I}$ with respect to the $2^{+}$of ${ }^{136} \mathrm{Te}$ core confirmed the similarity with its $A-2$ neighbor. Furthermore, it was noted in Ref. [43] that the level scheme of the ${ }^{139} \mathrm{Cs}$ nucleus, for example, is more uniform than that of the Xe partner, suggesting that the two extra protons in $\mathrm{Cs}$ induce more collective motion. An identical conclusion was given later in Ref. [22] concerning ${ }^{137}$ I with respect to the ${ }^{136} \mathrm{Te}$ nucleus, whose evenly distributed levels should also be caused by the extra proton-induced collectivity. Here, one can additionally compare the proton-induced collectivity with that induced by the increase of $N$. For example, from comparing the four-valence neutron ${ }^{139}$ I nucleus and the five-valence proton ${ }^{139} \mathrm{Cs}$ nucleus, it may be noticed that proton-induced collectivity looks weaker. It seems to induce a smaller compression in excited levels, and possibly implies relatively large orbital spacing than those seen in the iodine nuclei with the addition of neutrons. This would support the theoretical conclusions of Ref. [63], predicting a strong neutron dominance beyond $N=82$. Comparing the Te and Xe spectroscopic results, the authors concluded that the neutron-proton exchange symmetry breaking has a strong effect on excitation energies and transition rates of these nuclei.

\section{Transition rates}

Reduced transition probabilities listed in Table II are deduced from the measured lifetimes in this work. As stated 

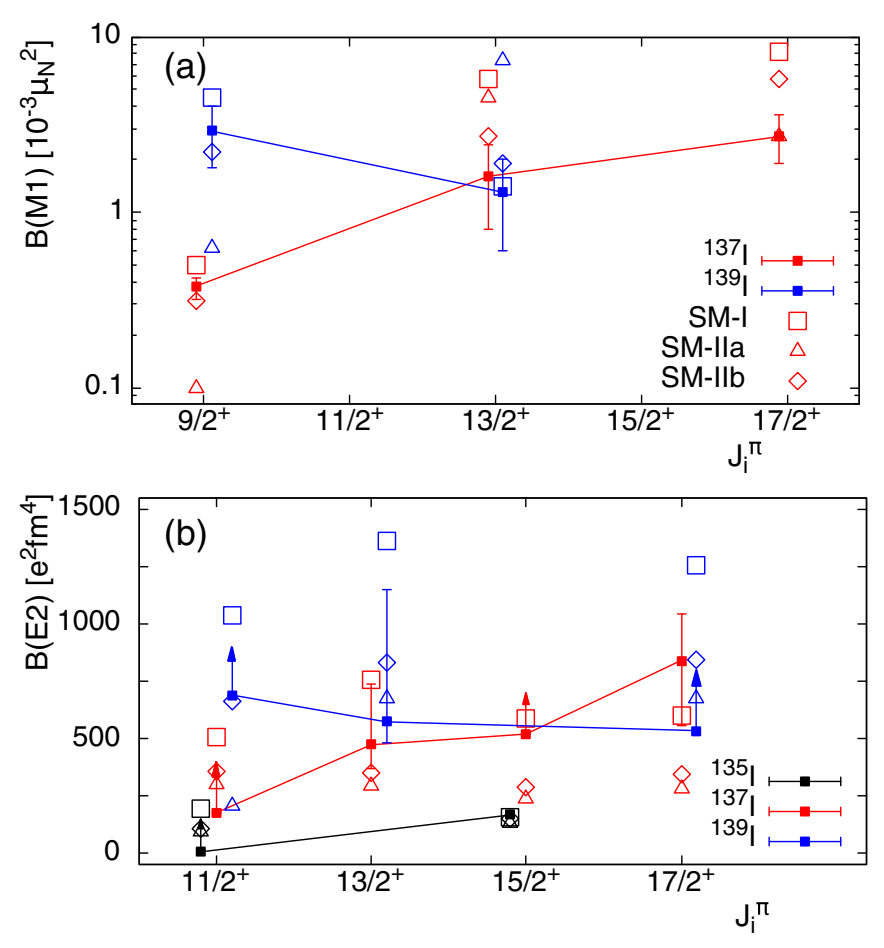

FIG. 11. Reduced transition probabilities given as (a) $B\left(M 1 ; J_{i} \rightarrow J_{i}-1\right)$ and (b) $B\left(E 2 ; J_{i} \rightarrow J_{i}-2\right)$. Experimental values are shown as filled squares and theoretical values from shell-model calculations as empty symbols. Solid lines for experimental values are drawn to guide the eye. All experimental values are determined in this work. The notation for the theoretical values is given in Table II.

in Sec. III D, for nonmixed $E 2$ transitions, $B(E 2)$ values are calculated taking into account their conversion [42], while for mixed $M 1+E 2$ transitions, in addition, respective mixing ratios are also considered [24,25]. For multiple transitions decaying from the same state, partial lifetimes are deduced using experimental intensity ratios given in Table I. Plotted in Fig. 11, the experimental transition rates are directly compared to those calculated within the framework of the shell model in our attempt to give insight into the nuclear wave functions of the connecting states.

\section{Specific B(E2) strengths}

In the ${ }^{135} \mathrm{I}$ isotope, the lifetime the $\left(15 / 2^{+}\right)$state $[\tau=$ $2340(115) \mathrm{ps}$ ] is consistent with the previous measurement [ $\tau=2510(120) \mathrm{ps}]$ [20]. The experimental $B(E 2)$ value of 168(9) $e^{2} \mathrm{fm}^{4}$ perfectly agrees with the results from the shell model of $157 e^{2} \mathrm{fm}^{4}$ provided by SM-I, though the results of SM-II are also reasonable. This corresponds to a value of 4.1(2) W.u., which is typical for semimagic nuclei. The $\left(15 / 2^{+}\right) \rightarrow\left(11 / 2^{+}\right)$transition rate in the ${ }^{135}$ I isotope is successfully reproduced in a seniority single- $j$ approach based on the $\pi\left(0 g_{7 / 2}^{3}\right)$ configuration and the $B\left(E 2 ; 4_{1}^{+} \rightarrow 2_{1}^{+}\right)$value of 4.30(39) W.u. in ${ }^{134} \mathrm{Te}$ [20]. From the experimental upper lifetime limit of $50 \mathrm{ps}$ for the $\left(11 / 2^{+}\right)$state in ${ }^{135} \mathrm{I}$ a lower $B(E 2)$ limit of $7 e^{2} \mathrm{fm}^{4}$ can be obtained. From the shell-model result, a lifetime of about 2 ps should be expected, which is below the sensitivity limit of the fast-timing technique.

The $\left(11 / 2^{+}\right)$states could be investigated in the heavier ${ }^{137,139} \mathrm{I}$ isotopes. The evolution of quadrupole collectivity inferred by the $B(E 2)$ strengths of the $\left(11 / 2^{+}\right) \rightarrow\left(7 / 2^{+}\right)$ ground state transition is comparable to the $2^{+} \rightarrow 0^{+}$in the even-even Te partners, where such an increase was also observed in our recent work [28]. Despite providing only lower limits here, based on the measured quantities, these transition probabilities show an indication of an increased collectivity with increasing valence nucleon number. Such a moderate change from $N=82\left({ }^{135} \mathrm{I}\right)$ toward $N=86\left({ }^{139} \mathrm{I}\right)$ is visible in Fig. 11, indicating a smooth development of collectivity properties outside the ${ }^{132} \mathrm{Sn}$ core. Also for these three-valence proton nuclei, the trend seems to be somewhat larger than the slowed down behavior seen in the two-proton Te [28], which can be expected. Compared to the theory one has to note that the slight increase of this collectivity is coherent with both SM-I and SM-II, even though somewhat overestimated. The trend is coherent with SM-IIa, which represents a different set of effective charges (see Sec. IV) and seems to account for the compression factor slightly better, while SM-IIb results have another tendency.

Several other states could be investigated, such as the $\left(13 / 2^{+}\right)$and the $\left(17 / 2^{+}\right)$, providing the opportunity to discuss the evolution of collectivity in terms of $B(E 2)$ and $B(M 1)$ strength with increasing neutron number, as can be seen in Table II. The $B(E 2)$ values suggest a slight increase in transition strength when moving from the $\left(11 / 2^{+}\right)$state to the $\left(13 / 2^{+}\right)$state in ${ }^{137} \mathrm{I}$. This trend is generally supported by all shell-model calculations. For the ${ }^{139} \mathrm{I}$ nucleus, a slight decrease from the $B\left(E 2 ;\left(11 / 2^{+}\right) \rightarrow\left(7 / 2^{+}\right)\right)$to the $B\left(E 2 ;\left(13 / 2^{+}\right) \rightarrow\left(9 / 2^{+}\right)\right)$strength could be seen (Fig. 11), which may be due to the uncertainty in the result, though also indicating a reduced trend with increasing $N$. As indicated in the Te isotones, this behavior is not well supported by the shell-model results, where with SM-I much larger quadrupole collectivity is predicted, while the trend with both interactions is the same independently of the effective charges. It is somewhat difficult to exactly reproduce the indicated collectivity by the experimental results for $A=139$. As the behavior of the dominantly $\pi\left(d_{5 / 2}\right)$-based $\left(17 / 2^{+}\right)$states is similar, one may conclude that both $\pi\left(d_{5 / 2}\right)$ and $\pi\left(g_{7 / 2}\right)$ orbitals, important in the $\left[\pi\left(0 g_{7 / 2}^{2} 1 d_{5 / 2}^{1}\right) v\left(1 f_{7 / 2}^{4}\right)\right]$ configurations of these states, contribute relatively equally to the decay strengths, while the obvious presence of reduced quadrupole collectivity is due to the larger excess of neutrons. This may be concluded by what is seen for $A=137$, where the experimental results are much better reproduced by the shell model, where SMI is best among those chosen for comparison. This is very well indicated in the comparison of $E 2$ transition strengths, especially for the $\left(13 / 2^{+}\right)$and $\left(17 / 2^{+}\right)$states, measured with reasonable uncertainty, but also for the $\left(15 / 2^{+}\right)$with a limit value deduced experimentally in our work. Thus, despite the relatively similar configurations in $A=137$ and $A=139$ (see Sec. IV A 2), the mixtures for the $N=86$ iodine nucleus do not seem to strongly influence an increase of collectivity. This is very interesting as our results provide a first direct measure of this $B(E 2)$ evolution for such very exotic 


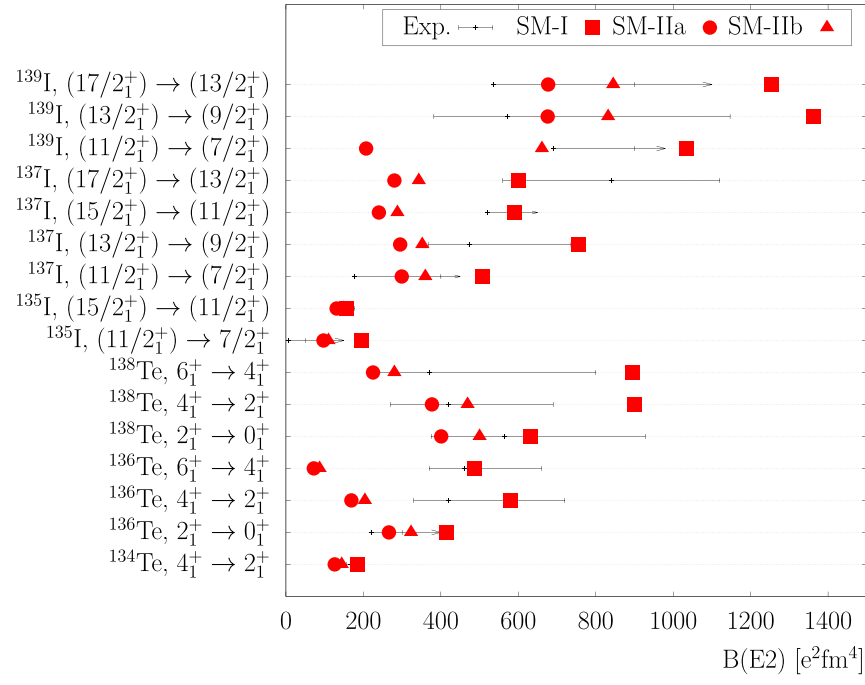

FIG. 12. E2 reduced transition probabilities of yrast transitions for neutron-rich tellurium and iodine isotopes beyond $N=82$. The data for ${ }^{134,136,138} \mathrm{Te}$ are taken from Ref. [28] and the results for the iodine isotopes are determined in this work.

species and, therefore, give an extremely valuable input to the extension of theoretical shell-model predictions for very neutron-rich nuclei. A graphical comparison of the experimental and theoretical $B(E 2)$ transition probabilities for these neutron-rich iodine isotopes and their respective tellurium isotones is shown in Fig. 12. The data for the tellurium isotopes are taken from Ref. [28] and further visualize the evolution collectivity, as discussed above.

As already indicated in Sec. IV A 2, the $B(E 2)$ transition strength of the higher-lying $\left(29 / 2^{+}\right) \rightarrow\left(25 / 2^{+}\right)$states can be reproduced only with the assumption of a relatively large hindrance factor. It is possible to account for this in the SM-II calculations, where values relatively close to the experimental ones are computed, and the effect of the effective charges is minor. Such a test was performed in Ref. [23] where the transition to the $\left(27 / 2^{+}\right)$state was also found to be hindered. It results in a theoretical value of $12 e^{2} \mathrm{fm}^{4}$ by SMI, compared to $18 e^{2} \mathrm{fm}^{4}$ [23] using $e_{\pi}=1.55 e, e_{\nu}=0.7 e$. The $B\left(E 2 ; 29 / 2^{+} \rightarrow 25 / 2^{+}\right)$transition predicted by SM-I is larger than the present measurement, whereas it is the same as that obtained by the SM calculations $\left(317 e^{2} \mathrm{fm}^{4}\right)$ reported in Ref. [23]. It is interesting to note that this transition represents a shared $\pi\left(d_{5 / 2}\right) \rightarrow \pi\left(g_{7 / 2}\right)$ and $\pi\left(d_{5 / 2}\right) \rightarrow \pi\left(d_{5 / 2}\right)$ strength as the $\left(29 / 2^{+}\right)$state is a very pure member of the $\pi\left(g_{7 / 2}^{2} d_{5 / 2}\right) v\left(f_{7 / 2}^{2}\right)$ multiplet. Here it appears to be connected to the $\left(25 / 2^{+}\right)$state which is about twice strongly fragmented.

\section{Specific B(M1) strengths}

The experimental $B(M 1)$ transition strengths are also shown in comparison to theory in Fig. 11 and Table II. The $B(M 1)$ values of the $\left(9 / 2^{+}\right) \rightarrow\left(7 / 2^{+}\right)$transition in ${ }^{139} \mathrm{I}$ is almost an order of magnitude higher than for its neighboring isotope with the difference of only two neutrons. As expected, this indicates an increase in collectivity when moving away from the closed shell. Although the main component of the $\left(9 / 2^{+}\right)$wave function is the same in both isotopes, the mixtures overtake the wave function in ${ }^{139} \mathrm{I}$ much more severely than in the other excited states with only about $5 \%$ of the main $\pi\left(g_{7 / 2}^{2} d_{5 / 2}\right) v\left(f_{7 / 2}^{4}\right)$ configuration.

For the $\left(13 / 2^{+}\right)$states no multipole mixing ratio is known for the mixed $(M 1+E 2)\left(13 / 2^{+}\right) \rightarrow\left(11 / 2^{+}\right)$transition and for ${ }^{139} \mathrm{I}$ only a lower limit is given. In ${ }^{137} \mathrm{I}$, a strong increase in $B(M 1)$ strength is indicated between the $J=9 / 2^{+}$and $J=13 / 2^{+}$states by almost an order of magnitude. Such a trend does not seem likely or as strongly pronounced for ${ }^{139} \mathrm{I}$, although no firm conclusions can be drawn from the lower $B(M 1)$ limit. Even for the known case, as the mixing is not severe, the $M 1$ strength is the dominating one and it clearly exhibits its collective behavior together with the evolution trend for the nuclei of interest.

Although the $g$ factor quenching of $0.7 g_{\text {free }}$ is typically used for this region [4,5] and performs relatively well, a test performed with another set is also possible, especially as this is directly related to the $B(M 1)$ strength. It shows that for the $\left(13 / 2^{+}\right)$states a $0.6 g_{\text {free }}$ quenching may be the better choice when using the SM-IIb that also drastically changes the order of magnitude for $\left(9 / 2^{+}\right) M 1$ strength in $A=137$ (e.g., to $10^{-4}$ ) and brings it closer to the experimental value. This provides a direct hint that reproducing the experimental evolution of collectivity is far from sufficient, and more experimental data should be collected for these very exotic systems.

\section{Specific B(E3) strengths}

Concerning the $B\left(E 3 ;\left(23 / 2^{-}\right) \rightarrow\left(17 / 2^{+}\right)\right)$transition rate in ${ }^{135} \mathrm{I}$ as stated in Sec. IV A, our experimental result is in agreement with the expectations of $8.6 \times 10^{3} e^{2} \mathrm{fm}^{6}$ from Ref. [44] based on a pure proton coupling to the equivalent state in ${ }^{134}$ Te with $B\left(E 3 ;\left(9^{-}\right) \rightarrow\left(6_{2}^{+}\right)\right)$of $8.7(14) \times 10^{3} e^{2} \mathrm{fm}^{6}$ [64]. Some agreement can also be found within the calculated $12.9 \times 10^{3} e^{2} \mathrm{fm}^{6}$ (11.96 W.u.) value quoted in the comparison with SM calculations of Ref. [65]. In this case also the Kuo-Herling interaction is used, though with a different set of single-particle energies and proton effective charge of $2.0 e$. It was concluded to be a good choice of the octupole effective charge for protons and the best to reproduce the observation of Ref. [64]. Based on this investigation it may be suggested that the measured $B(E 3)$ strengths in ${ }^{134} \mathrm{Te}$ (to both first and second excited $6^{+}$states, 3.8(2) and 8.0(1.3) W.u. respectively) $[64,65]$ show substantially low octupole collectivity above ${ }^{132} \mathrm{Sn}$ in comparison to other regions.

\section{SUMMARY}

In this work we have studied the deexcitation schemes of three odd-even ${ }^{[135,137,139]}$ I isotopes beyond ${ }^{132} \mathrm{Sn}$, providing the first lifetime data on iodine isotopes beyond $N=82$. We have reported whenever possible, e.g., due to lifetime range and/or poor statistics, first experimental outcomes on the highly demanded lifetimes of the first excited states in these nuclei, such as the $\left(9 / 2_{1}^{+}\right)$and $\left(13 / 2_{1}^{+}\right)$states in ${ }^{137} I$ and ${ }^{139} \mathrm{I}$, and the $\left(17 / 2_{1}^{+}\right)$state in ${ }^{137} \mathrm{I}$ isotopes. For several other states [the $\left(11 / 2_{1}^{+}\right)$states in ${ }^{135 c-139} \mathrm{I}$, the $\left(15 / 2_{1}^{+}\right)$state 
in ${ }^{137} \mathrm{I}$ and the $\left(17 / 2_{1}^{+}\right)$state in ${ }^{139} \mathrm{I}$, we have provided an upper lifetime limit that has also valuable indication. While the isomer data in ${ }^{135}$ I for the $\left(15 / 2_{1}^{+}\right)$and $\left(23 / 2_{1}^{-}\right)$states agrees well with single-proton coupling to the even-Te core, the new lifetime data on the $\left(29 / 2_{1}^{+}\right)$state in ${ }^{137}$ I confirms the previously suggested large $E 2$ hindrance at high-spin for $N=84$ and deserves special attention in future dedicated studies.

Inferred from the investigation of excited states, the deduced transition rates for mostly E2, $M 1$ (or mixed M1) transitions indicate with precision or give in several cases lower limits on the collectivity in these ${ }^{135,137,139} \mathbf{I}$ isotopes. Furthermore, in the comparison with state-of-theart shell-model calculations a reasonable agreement with the data could be seen, especially for the use of effective interactions, providing at the same time very valuable input for future theoretical works in the region. Interestingly, the behavior of the $B(E 2)$ strengths and the expected fast development of collectivity in these nuclei seems not to be indicated from the present data, in a more complex picture than expected. Moreover, the collectivity is slowed down significantly with the addition of neutrons. This is especially visible for the $\left(11 / 2_{1}^{+}\right)$states and in the $B(M 1)$ rates for the $\left(9 / 2_{1}^{+}\right)$states, although a non-negligible constant behavior is detected for the higher-lying $\left(13 / 2_{1}^{+}\right)$and $\left(15 / 2_{1}^{+}\right)$states examined in this work. Containing only a proton particle in addition, this trend in the iodine nuclei is not very different from their Te isotones and opens an interesting area for detailed studies of higher-lying states and on more exotic species around $N=90$.

In this fission reaction induced by fast neutrons on ${ }^{238} \mathrm{U}$, we have observed the population of angular momenta which were earlier accessed in spontaneous or ultracold neutron-induced fission of heavier fissioning elements, only. This observation remains intriguing, and may be of interest regarding the ques- tion of generation of spins in the fission fragments. Detecting relatively high spins similar to those previously known for these iodine nuclei, we provide complementary data useful for comparisons and further exploitation. It is compelling to continue these investigations with studies in fundamental nuclear physics and nuclear energy applications.

\section{ACKNOWLEDGMENTS}

The authors would like to thank the operators of the ALTO facility for providing the reliable beams used during the experiment. Additionally, we thank the FASTER Collaboration for the technical support given. GAMMAPOOL and LOANPOOL are acknowledged for loaning the clover and Phase-I HPGe detectors. The FATIMA Collaboration is acknowledged for loaning the $\mathrm{LaBr}_{3}(\mathrm{Ce})$ detectors. Some of the coauthors of this work were supported by the STFC UK Nuclear Data Network, the STFC (Grants No. ST/L005743/1 and No. ST/P005314), the Marion Redfearn Trust, BMBF (NUSTAR.DE Grants No. 05P15RDFN1 and No. 05P19PKFNA), and MINECO Grants No. FPA2015-65035-P and No. RTI2018-098868-B-I00. Part of the collaboration has received funding from the European Union's HORIZON 2020 Program under Grant Agreement No. 654002. G.H. and R.L. acknowledge support from the IDEX-API grant. P.H.R., S.M.C., M.B., and A.B. acknowledge support from the UK Government Department of BEIS via the National Measurement System, and the STFC consolidated grant. A.B., R.-B.G., and N.W. are supported by the DFG under Grant No. BL 1513/1-1. F.Z. received support from the Research Council of Norway under Contract No. 263030. M.P.-S. received funding from the Polish National Science Centre under Grants No. 2019/33/N/ST2/03023 and 2020/36/T/ST2/00547. A.K. was partially funded by Grant No. 2020/39/B/ST2/02346.
[1] J. Terasaki, J. Engel, W. Nazarewicz, and M. Stoitsov, Phys. Rev. C 66, 054313 (2002).

[2] N. Shimizu, T. Otsuka, T. Mizusaki, and M. Honma, Phys. Rev. C 70, 054313 (2004).

[3] L. Coraggio, A. Covello, A. Gargano, and N. Itaco, Phys. Rev. C 87, 034309 (2013).

[4] A. E. Stuchbery, J. M. Allmond, M. Danchev, C. Baktash, C. R. Bingham, A. Galindo-Uribarri, D. C. Radford, N. J. Stone, and C.-H. Yu, Phys. Rev. C 96, 014321 (2017).

[5] H. Naïdja, F. Nowacki, and B. Bounthong, Phys. Rev. C 96, 034312 (2017).

[6] J. M. Allmond, A. E. Stuchbery, C. Baktash, A. Gargano, A. Galindo-Uribarri, D. C. Radford, C. R. Bingham, B. A. Brown, L. Coraggio, A. Covello, M. Danchev, C. J. Gross, P. A. Hausladen, N. Itaco, K. Lagergren, E. Padilla-Rodal, J. Pavan, M. A. Riley, N. J. Stone, D. W. Stracener et al., Phys. Rev. Lett. 118, 092503 (2017).

[7] R. Lozeva, E. A. Stefanova, H. Naïdja, F. Nowacki, T. Rząca-Urban, J. Wisniewski, W. Urban, I. Ahmad, A. Blanc, G. De France, F. Didierjean, G. Duchêne, H. Faust, J. P. Greene, U. Köster, P. Mutti, G. Simpson, A. G. Smith,
T. Soldner, and C. A. Ur, Phys. Rev. C 98, 024323 (2018)

[8] H. Naïdja and F. Nowacki, EPJ Web Conf. 193, 01005 (2018).

[9] O. Artun, Int. J. Mod. Phys. E 27, 1850070 (2018).

[10] V. Paar, Phys. Lett. B 39, 466 (1972).

[11] G. Vanden Berghe, Z. Phys. 266, 139 (1974).

[12] R. A. Meyer, J. H. Landrum, S. V. Jackson, W. H. Zoller, and W. B. Walters, Phys. Rev. C 13, 1617 (1976).

[13] T. Rząca-Urban, K. Pągowska, W. Urban, A. Złomaniec, J. Genevey, J. A. Pinston, G. S. Simpson, M. Saha Sarkar, S. Sarkar, H. Faust, A. Scherillo, I. Tsekhanovich, R. Orlandi, J. L. Durell, A. G. Smith, and I. Ahmand, Phys. Rev. C 75, 054319 (2007).

[14] W. Urban, T. Rzaca-Urban, A. Korgul, J. L. Durell, M. J. Leddy, M. A. Jones, W. R. Phillips, A. G. Smith, B. J. Varley, I. Ahmad, L. R. Morss, and N. Schulz, Phys. Rev. C 65, 024307 (2002).

[15] R. Lozeva, H. Naïdja, F. Nowacki, J. Dudek, A. Odahara, C.-B. Moon, S. Nishimura, P. Doornenbal, J.-M. Daugas, P.-A. Söderström, T. Sumikama, G. Lorusso, J. Wu, Z. Y. Xu, H. Baba, F. Browne, R. Daido, Y. Fang, T. Isobe, I. Kojouharov et al., Phys. Rev. C 93, 014316 (2016). 
[16] P. Bhattacharyya, C. T. Zhang, B. Fornal, P. J. Daly, Z. W. Grabowski, I. Ahmad, T. Lauritsen, L. R. Morss, W. R. Phillips, J. L. Durell, M. J. Leddy, A. G. Smith, W. Urban, B. J. Varley, N. Schulz, E. Lubkiewicz, M. Bentaleb, and J. Blomqvist, Phys. Rev. C 56, R2363(R) (2008).

[17] B. Fogelberg, K. A. Mezilev, V. I. Isakov, K. I. Erokhina, H. Mach, E. Ramström, and H. Gausemel, Phys. Rev. C 75, 054308 (2007).

[18] W. Urban, M. Saha Sarkar, T. Rzaca-Urban, J. L. Durell, A. G. Smith, J. A. Genevey, J. A. Pinston, G. S. Simpson, and I. Ahmad, Eur. Phys. J. A 27, 257 (2006).

[19] B. Singh, A. A. Rodionov, and Y. L. Khazov, Nucl. Data Sheets 109, 517 (2008).

[20] P. Spagnoletti, G. S. Simpson, R. Carroll, J.-M. Régis, A. Blanc, M. Jentschel, U. Köster, P. Mutti, T. Soldner, G. de France, C. A. Ur, W. Urban, A. M. Bruce, F. Drouet, L. M. Fraile, L. P. Gaffney, D. G. Ghită, S. Ilieva, J. Jolie, W. Korten et al., Phys. Rev. C 95, 021302(R) (2017).

[21] M. Samri, G. J. Costa, G. Klotz, D. Magnac, R. Seltz, and J. P. Zirnheld, Z. Phys. A 321, 255 (1985).

[22] A. Korgul, W. Urban, T. Rzaca-Urban, M. Górska, J. L. Durell, M. J. Leddy, M. A. Jones, W. R. Phillips, A. G. Smith, B. J. Varley, M. Bentaleb, E. Lubkiewicz, N. Schulz, I. Ahmad, and L. R. Morss, Eur. Phys. J. A 12, 129 (2001).

[23] S. H. Liu, J. H. Hamilton, A. V. Ramayya, A. Covello, A. Gargano, N. Itaco, Y. X. Luo, J. O. Rasmussen, J. K. Hwang, A. V. Daniel, G. M. Ter-Akopian, S. J. Zhu, and W. C. Ma, Phys. Rev. C 80, 044314 (2009).

[24] E. Browne and J. K. Tuli, Nucl. Data Sheets 108, 2173 (2007).

[25] P. K. Joshi, B. Singh, S. Singh, and A. K. Jain, Nucl. Data Sheets 138, 1 (2016).

[26] B. Moon, C.-B. Moon, P.-A. Söderström, A. Odahara, R. Lozeva, B. Hong, F. Browne, H. S. Jung, P. Lee, C. S. Lee, A. Yagi, C. Yuan, S. Nishimura, P. Doornenbal, G. Lorusso, T. Sumikama, H. Watanabe, I. Kojouharov, T. Isobe, H. Baba et al., Phys. Rev. C 95, 044322 (2017).

[27] J.-M. Régis, H. Mach, G. S. Simpson, J. Jolie, G. Pascovici, N. Saed-Samii, N. Warr, A. Bruce, J. Degenkolb, L. M. Fraile, C. Fransen, D.G. Ghita, S. Kisyov, U. Köster, A. Korgul, S. Lalkovski, N. Mărginean, P. Mutti, B. Olaizola, Z. Podolyak et al., Nucl. Instrum. Methods Phys. Res., Sect. A 726, 191 (2013).

[28] G. Häfner, R. Lozeva, H. Nadïja, M. Lebois, N. Jovančević, D. Thisse, D. Etasse, R. L. Canavan, M. Rudigier, J. N. Wilson, E. Adamska, P. Adsley, M. Babo, K. Belvedere, J. Benito, G. Benzoni, A. Blazhev, A. Boso, S. Bottoni, M. Bunce et al., Phys. Rev. C 103, 034317 (2021).

[29] G. Locatelli, M. Mancini, and N. Todeschini, Energy Policy 61, 1503 (2013).

[30] http://world-nuclear.org (2021).

[31] M. Lebois, J. N. Wilson, P. Halipre, B. Leniau, I. Matea, A. Oberstedt, S. Oberstedt, and D. Verney, Nucl. Instrum. Methods Phys. Res., Sect. A 735, 145 (2014).

[32] R.-B. Gerst, A. Blazhev, N. Warr, J. N. Wilson, M. Lebois, N. Jovančević, D. Thisse, R. Canavan, M. Rudigier, D. Étasse, E. Adamska, P. Adsley, A. Algora, M. Babo, K. Belvedere, J. Benito, G. Benzoni, A. Boso, S. Bottoni, M. Bunce et al., Phys. Rev. C 102, 064323 (2020).

[33] M. Lebois, N Jovančević, D. Thisse, R. Canavan, D. Étasse, M. Rudigier, and J. N. Wilson, Nucl. Instrum. Methods Phys. Res., Sect. A 960, 163580 (2020).
[34] S. Agostinelli, J. Allison, K. Amako, J. Apostolakis, H. Araujo, P. Arce, M. Asai, D. Axen, S. Banerjee, G. Barrand, F. Behner, L. Bellagamba, J. Boudreau, L. Broglia, A. Brunengo, H. Burkhardt, S. Chauvie, J. Chuma, R. Chytracek, G. Cooperman et al., Nucl. Instrum. Methods Phys. Res., Sect. A 506, 250 (2003)

[35] http://faster.in2p3.fr (2021).

[36] G. Häfner, R. Lozeva, A. Blazhev, R. L. Canavan, D. Etasse, J. Jolie, N. Jovancevic, R.-B. Gerst, Th. Kröll, M. Lebois, P. H. Regan, M. Rudigier, D. Thisse, N. Warr, J. N. Wilson, and the N-SI-109 Collaboration, J. Phys.: Conf. Ser. 1643, 012135 (2020).

[37] J. N. Wilson, M. Lebois, L. Qi, P. Amador-Celdran, D. Bleuel, J. A. Briz, R. Carroll, W. Catford, H. De Witte, D. T. Doherty, R. Eloirdi, G. Georgiev, A. Gottardo, A. Goasduff, K. Hadyńska-Klęk, K. Hauschild, H. Hess, V. Ingeberg, T. Konstantinopoulos, J. Ljungvall et al., Phys. Rev. Lett. 118, 222501 (2017).

[38] J. Katakura, JAEA-Data/Code, Japan Atomic Energy Agency, 2011.

[39] J. Katakura, F. Minato, and K. Ohgama, EPJ Web Conf. 111, 08004 (2016)

[40] J. N. Wilson, D. Thisse, M. Lebois, N. Jovancevic, D. Gjestvang, R. Canavan, M. Rudigier, D. Étasse, R.-B. Gerst, L. Gaudefroy, E. Adamska, P. Adsley, A. Algora, M. Babo, K. Belvedere, J. Benito, G. Benzoni, A. Blazhev, A. Boso, S. Bottoni et al., Nature (London) 590, 566 (2021).

[41] S. K. Saha, C. Constantinescu, P. J. Daly, P. Bhattacharyya, C. T. Zhang, Z. W. Grabowski, B. Fornal, R. Broda, I. Ahmad, D. Seweryniak, I. Wiedenhöver, M. P. Carpenter, R. V. F. Janssens, T. L. Khoo, T. Lauritsen, C. J. Lister, and P. Reiter, Phys. Rev. C 65, 017302 (2001).

[42] T. Kibédi, T. W. Burrows, M. B. Trzhaskovskaya, P. M. Davidson, and C. W. Nestor, Jr., Nucl. Instrum. Methods Phys. Res., Sect. A 589, 202 (2008).

[43] C. T. Zhang, P. Bhattacharyya, P. J. Daly, R. Broda, Z. W. Grabowski, D. Nisius, I. Ahmad, T. Ishii, M. P. Carpenter, L. R. Morss, W. R. Phillips, J. L. Durell, M. J. Leddy, A. G. Smith, W. Urban, B. J. Varley, N. Schulz, E. Lubkiewicz, M. Bentaleb, and J. Blomqvist, Phys. Rev. Lett. 77, 3743 (1996).

[44] M.W. Simon, Ph.D. thesis, University of Rochester, 1999 (unpublished).

[45] Yu. Khazov, A. Rodionov, and F. G. Kondev, Nucl. Data Sheets 112, 855 (2011).

[46] W. Urban, W. Kurcewicz, A. Nowak, T. Razca-Urban, J. L. Durell, M. J. Leddy, M. A. Jones, W. R. Phillips, A. G. Smith, B. J. Varley, M. Bentaleb, E. Lubkiewciz, N. Schulz, J. Blomqvist, P. J. Daly, P. Bhattacharyya, C. T. Zhang, I. Ahmad, and L. R. Morss, Eur. Phys. J. A 5, 239 (1999).

[47] F. Andreozzi, L. Coraggio, A. Covello, A. Gargano, T. T. S. Kuo, and A. Porrino, Phys. Rev. C 56, R16(R) (1997).

[48] H. Naïdja, F. Nowacki, B. Bounthong, M. Czerwinski, T. RzacaUrban, T. Roginski, W. Urban, J. Wisniewski, K. Sieja, A. G. Smith, J. F. Smith, G. S. Simpson, I. Ahmad, and J. P. Greene, Phys. Rev. C 95, 064303 (2017).

[49] E. Caurier, G. Martínez-Pinedo, F. Nowack, A. Poves, and A. P. Zuker, Rev. Mod. Phys. 77, 427 (2005).

[50] E. Caurier and F. Nowacki, Acta Phys. Pol. B 30, 705 (1999).

[51] B. A. Brown and W. D. M. Rae, Nucl. Data Sheets 120, 115 (2014).

[52] J. B. McGrory and T. T. S. Kuo, Nucl. Phys. A 247, 283 (1975). 
[53] E. K. Warburton and B. A. Brown, Phys. Rev. C 43, 602 (1991).

[54] L. Rydström, J. Blomqvist, R. J. Liotta, and C. Pomar, Nucl. Phys. A 512, 217 (1990).

[55] N. Cieplicka, K. H. Maier, B. Fornal, B. Szpak, R. V. F. Janssens, M. Alcorta, R. Broda, M. P. Carpenter, C. J. Chiara, C. R. Hoffman, B. P. Kay, F. G. Kondev, W. Królas, T. Lauritsen, C. J. Lister, E. A. McCutchan, T. Pawłat, A. M. Rogers, D. Seweryniak, N. Sharp et al., Phys. Rev. C 86, 054322 (2012).

[56] Chong Qi, L. Y. Jia, and G. J. Fu, Phys. Rev. C 94, 014312 (2016).

[57] N. Lalovic, D. Rudolph, Zs. Podolyak, L. G. Sarmiento, E. C. Simpson, T. Alexander, M. L. Cortes, J. Gerl, P. Golubev, F. Ameil, T. Arici, Ch. Bauer, D. Bazzacco, M. A. Bentley, P. Boutachkov, M. Bowry, C. Fahlander, A. Gadea, J. Gellanki, A. Givechev et al., J. Phys. G: Nucl. Part. Phys. 45, 035105 (2018).

[58] A. Korgul, W. Urban, T. Rząca-Urban, M. Rejmund, J. L. Durell, M. J. Leddy, M. A. Jones, W. R. Phillips, A. G. Smith, B. J. Varley, N. Schulz, M. Bentaleb, E. Lubkiewicz, I. Ahmad, and L. R. Morss, Eur. Phys. J. A 7, 167 (2000).

[59] M. N. Mineva, M. Hellström, M. Bernas, J. Gerl, H. Grawe, M. Pfützner, P. H. Regan, M. Rejmund, D. Rudolph,
F. Becker, C. R. Bingham, T. Enqvist, B. Fogelberg, H. Gausemel, H. Geissel, J. Genevey, M. Górska, R. Grzywacz, K. Hauschild, Z. Janas et al., Eur. Phys. J. A 11, 9 (2001).

[60] J. Shergur, A. Wöhr, W. B. Walters, K.-L. Kratz, O. Arndt, B. A. Brown, J. Cederkall, I. Dillmann, L. M. Fraile, P. Hoff, A. Joinet, U. Köster, and B. Pfeiffer, Phys. Rev. C 71, 064321 (2005).

[61] R. Lozeva, A. Odahara, C.-B. Moon, S. Nishimura, P. Doornenbal, H. Naïdja, F. Nowacki, P.-A. Söderström, T. Sumikama, G. Lorusso, J. Wu, Z. Y. Xu, H. Baba, F. Browne, R. Daido, J.-M. Daugas, F. Didierjean, Y. Fang, T. Isobe, I. Kojouharov et al., Phys. Rev. C 92, 024304 (2015).

[62] C. Goodin, N. J. Stone, A. V. Ramayya, A. V. Daniel, J. R. Stone, J. H. Hamilton, K. Li, J. K. Hwang, Y. X. Luo, J. O. Rasmussen, A. Gargano, A. Covello, and G. M. Ter-Akopian, Phys. Rev. C 78, 044331 (2008).

[63] D. Bianco, N. Lo Iudice, F. Andreozzi, A. Porrino, and F. Knapp, Phys. Rev. C 88, 024303 (2013).

[64] J. P. Omtvedt, H. Mach, B. Fogelberg, D. Jerrestam, M. Hellström, L. Spanier, K. I. Erokhina, and V. I. Isakov, Phys. Rev. Lett. 75, 3090 (1995).

[65] S. Sarkar and M. S. Sarkar, Phys. Rev. C 64, 014312 (2001). 